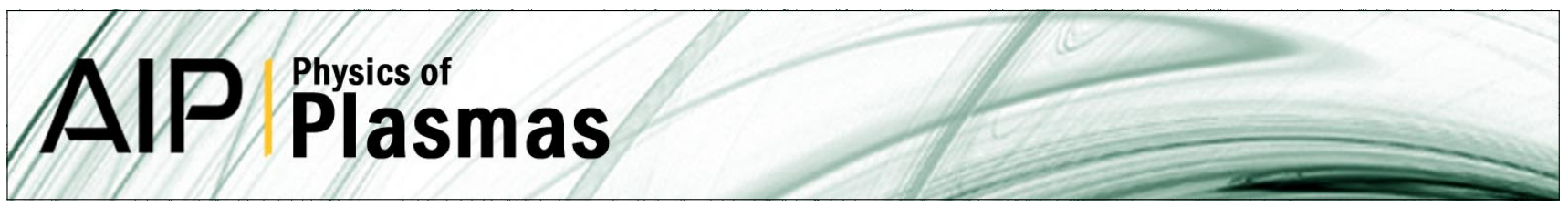

\title{
Toroidal modeling of interaction between resistive wall mode and plasma flow
}

Yueqiang Liu and Youwen Sun

Citation: Phys. Plasmas 20, 022505 (2013); doi: 10.1063/1.4793449

View online: http://dx.doi.org/10.1063/1.4793449

View Table of Contents: http://pop.aip.org/resource/1/PHPAEN/v20/i2

Published by the American Institute of Physics.

\section{Related Articles}

Boundary induced amplification and nonlinear instability of interchange modes Phys. Plasmas 20, 020704 (2013)

Generalized shock conditions and the contact discontinuity in the Hall-magnetohydrodynamics model Phys. Plasmas 20, 022112 (2013)

Comparison between hybrid and fully kinetic models of asymmetric magnetic reconnection: Coplanar and guide field configurations

Phys. Plasmas 20, 022902 (2013)

Generating vorticity and magnetic fields in plasmas in general relativity: Spacetime curvature drive

Phys. Plasmas 20, 022901 (2013)

Global current profile effects on the evolution and saturation of magnetic islands

Phys. Plasmas 20, 020702 (2013)

\section{Additional information on Phys. Plasmas}

Journal Homepage: http://pop.aip.org/

Journal Information: http://pop.aip.org/about/about_the_journal

Top downloads: http://pop.aip.org/features/most_downloaded

Information for Authors: http://pop.aip.org/authors

\section{ADVERTISEMENT}

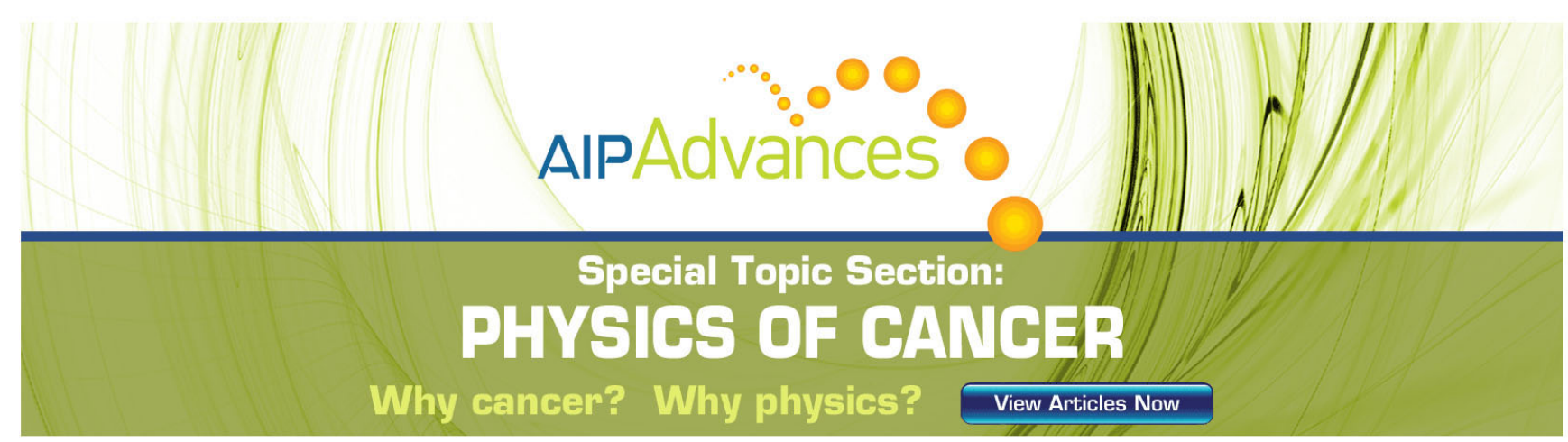




\title{
Toroidal modeling of interaction between resistive wall mode and plasma flow
}

\author{
Yueqiang Liu ${ }^{1, a)}$ and Youwen Sun ${ }^{2}$ \\ ${ }^{1}$ Euratom/CCFE Fusion Association, Culham Science Centre, Abingdon OX14 3DB, United Kingdom \\ ${ }^{2}$ Institute of Plasma Physics, Chinese Academy of Sciences, PO Box 1126, Hefei 230031, China
}

(Received 29 November 2012; accepted 31 January 2013; published online 21 February 2013)

The non-linear interplay between the resistive wall mode (RWM) and the toroidal plasma flow is numerically investigated in a full toroidal geometry, by simultaneously solving the initial value problems for the $n=1 \mathrm{RWM}$ and the $n=0$ toroidal force balance equation. Here, $n$ is the toroidal mode number. The neoclassical toroidal viscous torque is identified as the major momentum sink that brakes the toroidal plasma flow during the non-linear evolution of the RWM. This holds for a mode that is initially either unstable or stable. For an initially stable RWM, the braking of the flow, and hence the eventual growth of the mode, depends critically on the initial perturbation amplitude. [http://dx.doi.org/10.1063/1.4793449]

\section{INTRODUCTION}

The resistive wall mode (RWM) is a global, macroscopic magnetohydrodynamic (MHD) instability that often limits the operational space of fusion devices. ${ }^{1}$ The origin of the mode is often an equilibrium current or pressure driven external ideal kink instability. It is of particular importance to study the RWM physics and control in the so called advanced tokamaks, which aim at achieving economic fusion reactors. In advanced tokamaks, suppression of the RWM often leads to a significant gain in the plasma pressure under steady state conditions, and thus a significant gain in the eventual fusion power production.

Even though the RWM is often regarded, and in fact studied, both in theory and experiments, ${ }^{1}$ as a linear MHD mode, there is an abundant experimental evidence pointing to the non-linear interactions between this instability and other MHD modes, ${ }^{2}$ energetic particles, ${ }^{3,4}$ as well as the plasma flow. ${ }^{5}$ This work investigates the last question, namely, the nonlinear coupling between the RWM and the toroidal rotation of a tokamak plasma.

This question has previously been addressed in analytic theory, based on cylindrical approximations. ${ }^{6-8}$ For instance, in Ref. 6, a simple set of non-linear evolution equations is derived and investigated for the RWM in a large aspect ratio, rotating, viscous tokamak plasma. This model essentially couples the Fitzpatrick-Aydemir dispersion relation ${ }^{9}$ for a RWM to a toroidal momentum balance equation. Simulations in Ref. 6 show that a sufficiently large amplitude of the RWM triggers the plasma deceleration and the loss of the wall stabilization for the mode. A somewhat different RWM model (visco-resistive Finn mode ${ }^{10}$ ) was adopted in Ref. 7, which essentially investigated the non-linear interaction between an unstable resistive wall tearing mode (RWTM) and the plasma flow, with an additional presence of an external error field.

In this work, we carry out toroidal simulations of the non-linear coupling between the RWM and the plasma flow,

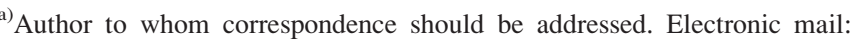
yueqiang.liu@ccfe.ac.uk.
}

using the recently developed MARS-Q code. ${ }^{11}$ Our RWM model follows a single fluid, full resistive MHD formulation in a generic toroidal geometry, with additional damping of the mode from a strong parallel sound wave damping model. We solve the MHD equations together with a toroidal momentum balance equation, which includes both the electromagnetic torque and the neoclassical toroidal viscous torque, induced by the $3 \mathrm{D}$ field perturbations due to a RWM. For simplicity, we neglect the additional effect from error fields, although the latter can be easily included into the MARS-Q formulation.

The toroidal formulation is introduced in Sec. II. Section III reports numerical results, where the non-linear interaction between an either unstable or a stable RWM, and the plasma flow is investigated. Section IV summarizes the work.

\section{TOROIDAL FORMULATION OF NON-LINEAR COUPLING BETWEEN RWM AND PLASMA FLOW}

The formulation is essentially the same as that of the MARS-Q code, ${ }^{11}$ which was developed for investigating the rotational braking and the penetration of resonant magnetic perturbation (RMP) fields into a stable plasma. Below, we give a brief description of the equations that we solve for studying the non-linear interaction between the RWM and the plasma flow, in a generic toroidal geometry. The RWM is described by single fluid perturbed MHD equations that incorporates a (sheared) toroidal flow, with the angular velocity $\Omega(r)$ that depends on the plasma minor radius $r$

$$
\begin{gathered}
\left(\frac{\partial}{\partial t}+i n \Omega\right) \xi=\mathbf{v}+(\xi \cdot \nabla \Omega) R^{2} \nabla \phi \\
\rho\left(\frac{\partial}{\partial t}+i n \Omega\right) \mathbf{v}=-\nabla p+\mathbf{j} \times \mathbf{B}+\mathbf{J} \times \mathbf{b} \\
-\rho\left[2 \Omega \nabla Z \times \mathbf{v}+(\mathbf{v} \cdot \nabla \Omega) R^{2} \nabla \phi\right]-\nabla \cdot \Pi
\end{gathered}
$$




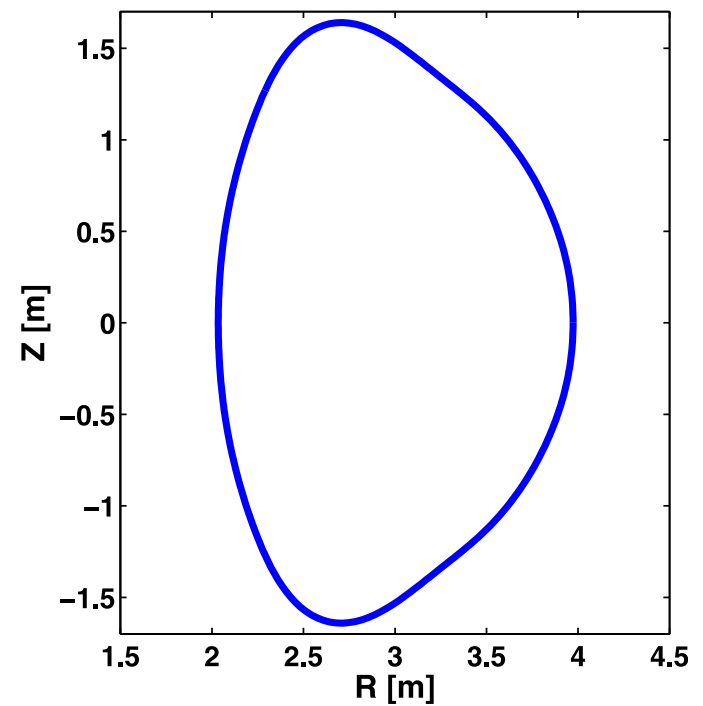

FIG. 1. The plasma boundary shape of the toroidal equilibrium.

$$
\begin{aligned}
\left(\frac{\partial}{\partial t}+i n \Omega\right) \mathbf{b} & =\nabla \times(\mathbf{v} \times \mathbf{B})+(\mathbf{b} \cdot \nabla \Omega) R^{2} \nabla \phi-\nabla \times(\eta \mathbf{j}) \\
\left(\frac{\partial}{\partial t}+i n \Omega\right) p & =-\mathbf{v} \cdot \nabla P-\Gamma P \nabla \cdot \mathbf{v}
\end{aligned}
$$

where $(R, Z, \phi)$ is the cylindrical coordinate system for the torus. The quantities $\rho, \mathbf{B}, \mathbf{J}=\nabla \times \mathbf{B}$, and $P$ denote the equilibrium plasma density, the magnetic field, the plasma current density, and the plasma pressure, respectively. The quantities $\xi, \mathbf{v}, \mathbf{b}, \mathbf{j}=\nabla \times \mathbf{b}$, and $p$, which are also our solution variables, represent the plasma displacement, the perturbed velocity, the magnetic field, the current, and the pressure, respectively. $n$ is the toroidal mode number. Note that, in this work, we consider only a single $n(n=1)$ RWM. This justifies the choice of the perturbed MHD equations. In the single fluid MHD approximation, the ratio of specific heats, $\Gamma$ from Eq. (4), is taken to be $5 / 3$ for an ideal gas. The above system
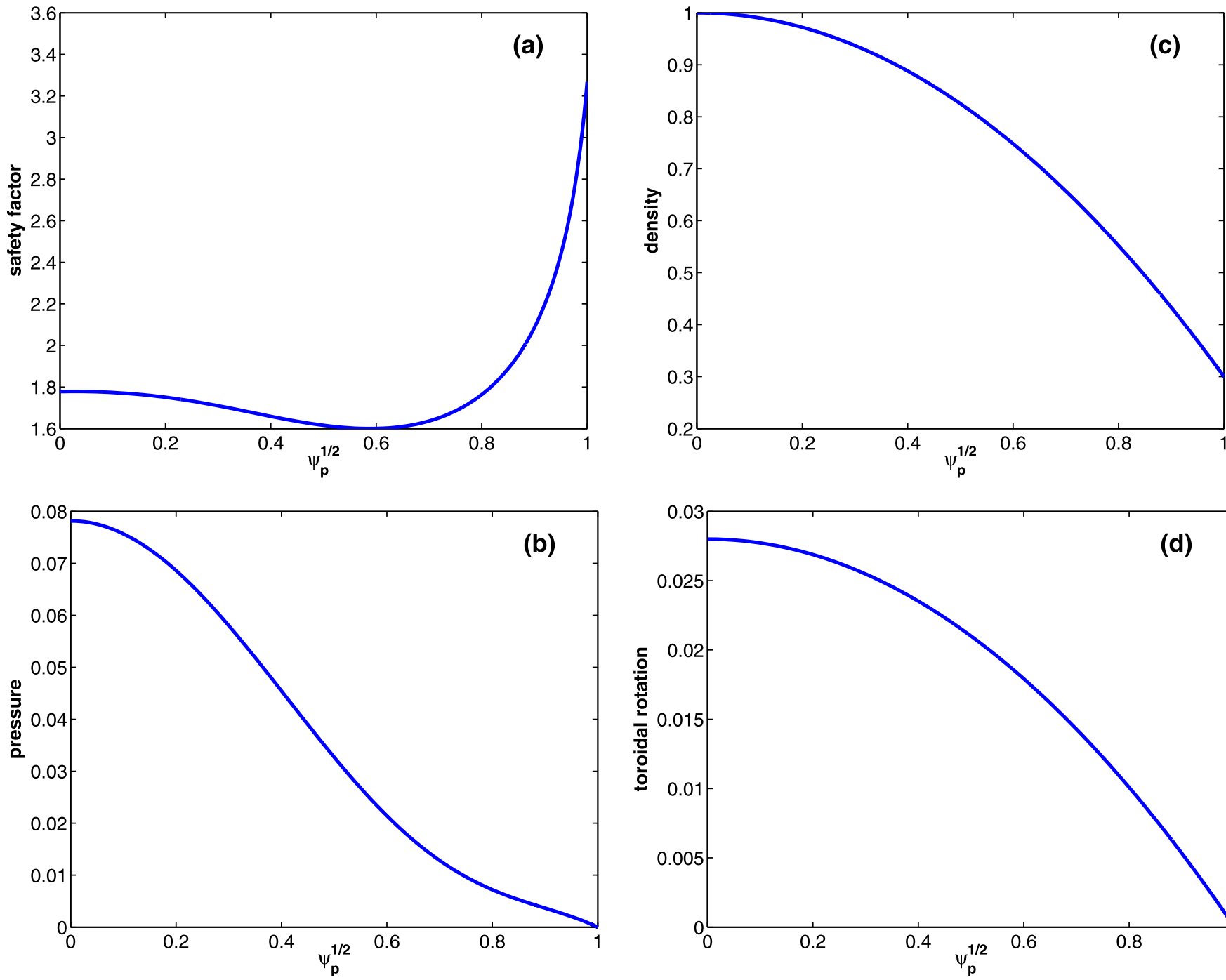

FIG. 2. Radial profiles of the equilibrium quantities for (a) the safety factor $q$, (b) the plasma pressure normalized by $B_{0}^{2} / \mu_{0}$, (c) the plasma density normalized to unity at the magnetic axis, and (d) the toroidal rotation frequency of the plasma, normalized by the on-axis Alfvén frequency. 
of equations is written and solved in a dimensionless form, where the time is normalized by the Alfvén time at the magnetic axis, the length is normalized by the major radius $R_{0}$ of the torus, the magnetic field is normalized by the toroidal vacuum field $B_{0}$ at the magnetic axis, and the pressure is normalized by $B_{0}^{2} / \mu_{0}$, where $\mu_{0}=4 \pi \times 10^{-7} \mathrm{H} / \mathrm{m}$ is the vacuum permeability.

It is worthwhile to comment on two specific components from the above model for the RWM. A damping term, in the form of a viscous tensor $\Pi$, is included into the momentum Eq. (2). For a proper description of the damping physics of the RWM, it is essential to include this type of extra damping terms. In principle, ideal MHD can provide continuum damping on the mode, both from Alfvén and sound waves. The former requires the existence of rational surfaces inside the plasma, whilst the latter occurs only in a finite pressure plasma. However, ideal MHD theory generally does not seem to adequately predict the mode damping observed in experiments, both on the critical rotation velocity for the mode suppression ${ }^{12}$ and on the resonant field amplification from the response of a stable RWM. ${ }^{13}$ So far, probably the most adequate damping model for the RWM is the drift kinetic model, in which the viscous tensor term is calculated from the drift kinetic theory, and represents the perturbed kinetic pressure tensor. ${ }^{14}$ On the other hand, it has been noted that, in many cases, the RWM damping can be modeled by a (strong) parallel viscous force ${ }^{15,16}$

$$
\nabla \cdot \Pi=\rho \kappa_{\|}\left|k_{\|} v_{t h, i}\right|\left[\mathbf{v}+(\xi \cdot \nabla) R^{2} \Omega \nabla \phi\right]_{\|},
$$

where $\kappa$ is a numerical coefficient determining the damping "strength." $k_{\|}=(n-m / q) / R$ is the parallel wave number, with $m$ being the poloidal harmonic number and $q$ being the safety factor. $v_{t h, i}=\sqrt{2 T_{i} / M_{i}}$ is the thermal ion velocity with $T_{i}, M_{i}$ being the thermal ion temperature and mass, respectively. The parallel component of the perturbed velocity is taken along the equilibrium field line. This damping model, originally coming from a fluid closure of the ion Landau damping of the parallel sound wave, is often used in the RWM modeling ${ }^{15,16}$ and in many cases provides adequate damping on the mode. This damping model is used in the present work.

As for the second comment, we use a resistive plasma model to describe the RWM in this study, as evident from Eq. (3), where $\eta$ is the plasma resistivity. With the normalization convention adopted in our formulation, $\eta$ is the inverse of the (magnetic) Lundquist number. The reason for choosing a resistive plasma is to improve the numerical resolution for the electromagnetic torque, which generally occurs near rational surfaces. A resistive model, with large Lundquist number at least in the plasma core, is also more relevant to realistic experiments. We point out that the electromagnetic torque can remain finite even in an ideal plasma. $^{17,18}$

Normally, the RWM is well described by ideal MHD (with extra damping physics as mentioned above). Inclusion of the plasma resistivity can have two effects on the RWM: It generally increases the mode growth rate; and more qualitatively, it couples the RWM to a tearing mode. ${ }^{10,19}$ The question of which mode becomes more dominant depends on the ratio between the plasma resistive time and the wall resistive time, among other physical parameters. In this work, we assume that the wall resistivity is much larger than the plasma resistivity (the plasma is close to an ideal plasma in the core), so that the mode remains a predominantly RWM.

For the RWM modeling, the above MHD equations inside the plasma are solved together with the vacuum equations outside the plasma, as well as a resistive wall equation with the thin shell approximation.

In addition, a toroidal momentum balance equation

$$
\frac{\partial L}{\partial t}=D(L)+T_{j \times b}+T_{\mathrm{NTV}}+T_{\text {source }},
$$

is also solved for $L=\rho\left\langle R^{2}\right\rangle \Omega$, where $\langle\cdot\rangle$ denotes the surface average of a quantity. $D(L)$ is a (linear) momentum diffusion operator. $T_{j \times b}$ is the surface averaged, toroidal electromagnetic $\mathbf{j} \times \mathbf{b}$ torque, computed as
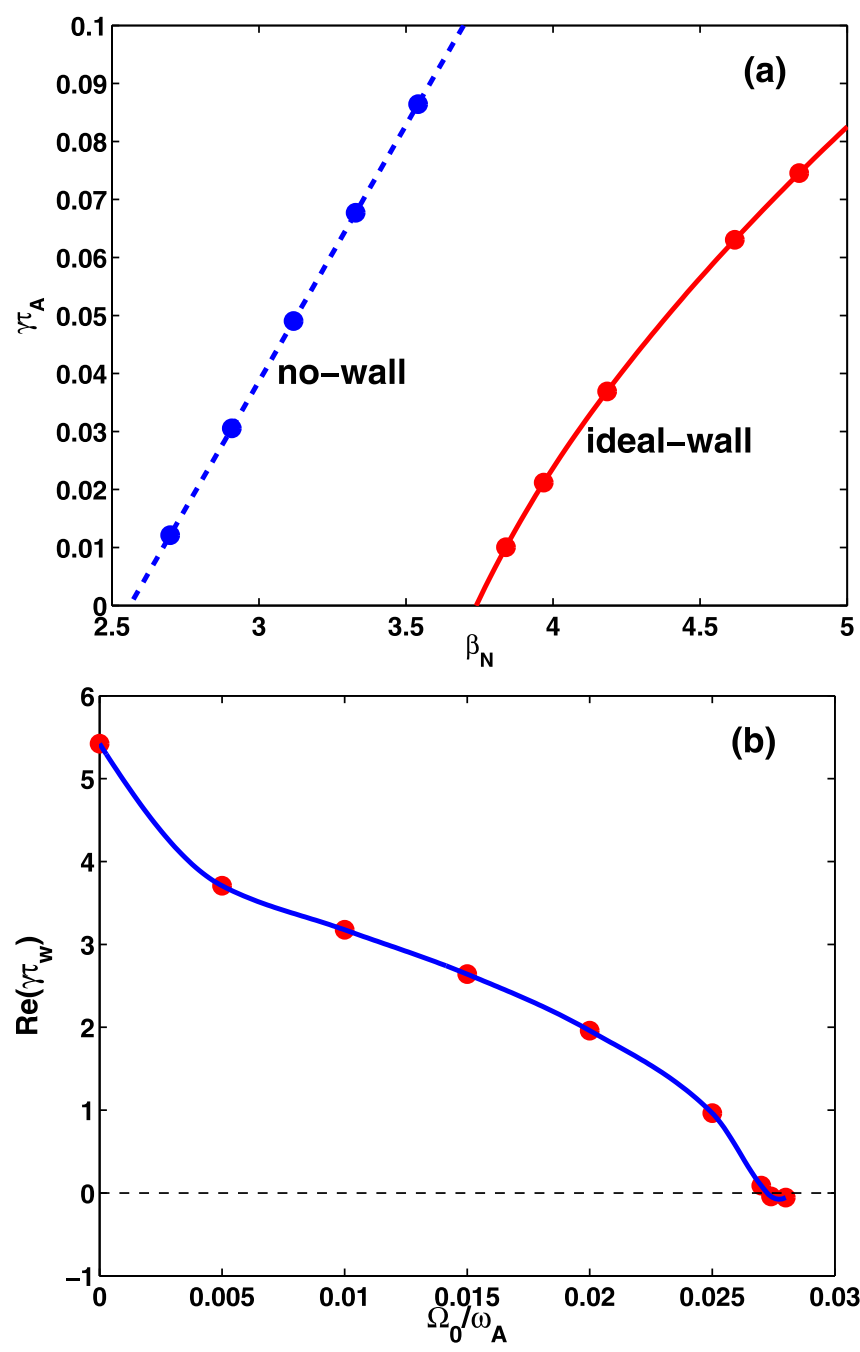

FIG. 3. Linear stability of (a) the ideal external, pressure-driven kink mode and (b) the resistive wall mode in the presence of the plasma flow. 


$$
T_{j \times b}=\oint R \mathbf{j} \times \mathbf{b} \cdot \hat{\phi} d S / \oint d S,
$$

where $R$ is the major radius, $\mathbf{j}$ and $\mathbf{b}$ are the (total) perturbed plasma current and magnetic field, respectively, as the solution of Eqs. (2)-(4). $S$ denotes the flux surface.

$T_{\mathrm{NTV}}$ is the neoclassical toroidal viscous (NTV) torque, computed using formulas from Ref. 20, where various regimes are smoothly connected. The module of the perturbed magnetic field (the Lagrangian part), from Eqs. (2) to (4), directly enters into the NTV computations. $T_{\text {source }}$ is the momentum source term. The RWM perturbations enter into the momentum sinks ( $T_{j \times b}$ and $\left.T_{\mathrm{NTV}}\right)$ in a quadratic form. The NTV torque, which is a consequence of the radial drift of banana orbits of trapped thermal particles, due to the presence of $3 \mathrm{D}$ magnetic fields, is generally a rather non-linear function of the plasma flow velocity. We refer to
Ref. 11 for detailed description of these sink and source terms in the MARS-Q formulation.

Note that the parallel viscous force, Eq. (5) which describes the damping of the $n \neq 0$ RWM, does not explicitly enter into the $n=0$ momentum balance equation (6).

An important point is that we assume that a momentum balance is reached at the start of our simulation $t=0$, such that $D(L(t=0))+T_{\text {source }}=0$. This condition essentially defines the initial rotation frequency. By further assuming that the momentum source does not change during the non-linear simulation, we instead solve the momentum balance equation for the change of the toroidal momentum, $\Delta L=L(t)-L(0)$,

$$
\frac{\partial \Delta L}{\partial t}=D(\Delta L)+T_{j \times b}+T_{N T V},
$$
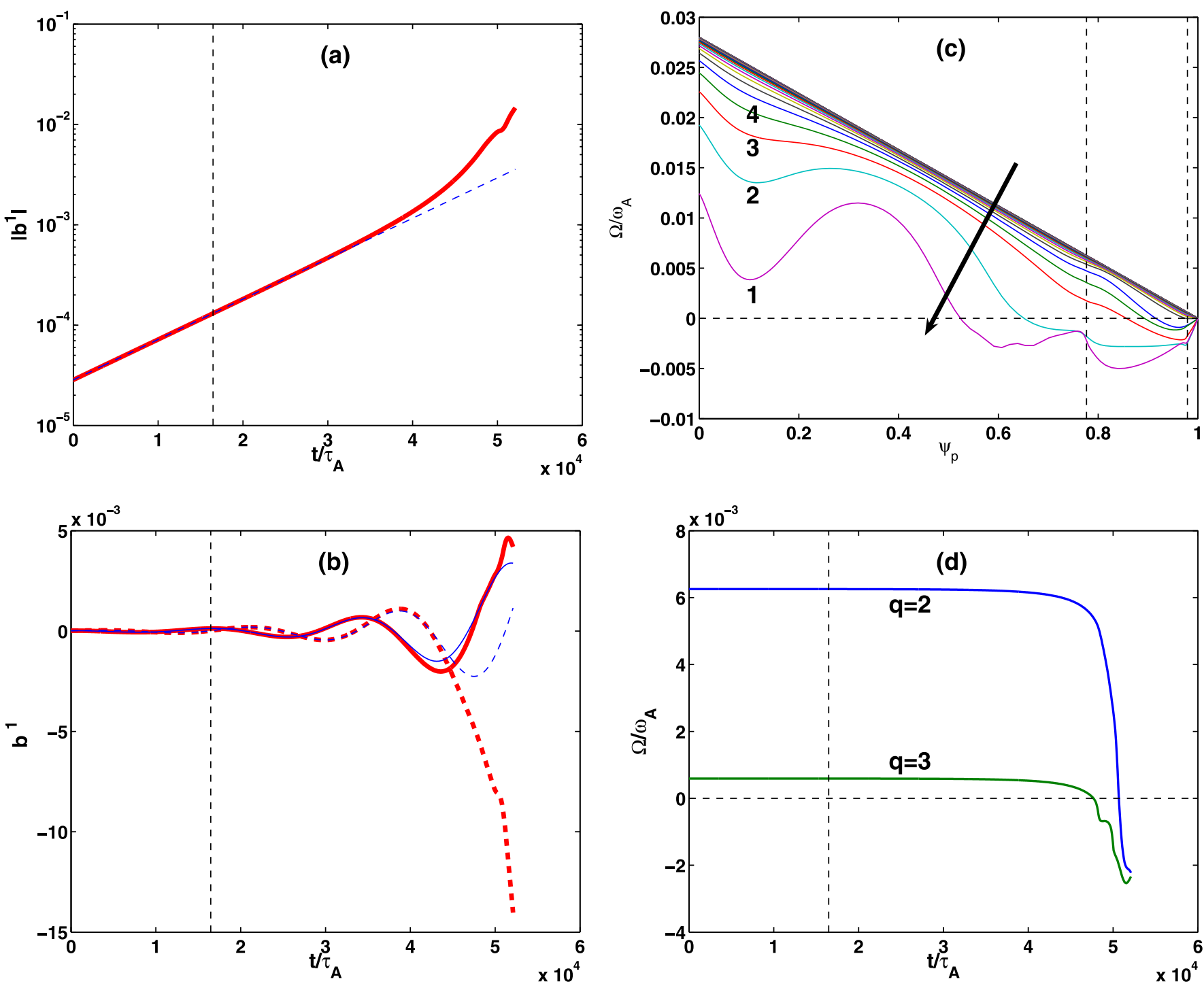

FIG. 4. Evolution of an initially unstable RWM: (a) the amplitude of the perturbed radial field $b^{1}$ at the $q=2$ surface, (b) the real and imaginary parts of the perturbed radial field $b^{1}$ at the $q=2$ surface, (c) the radial profile of the plasma rotation frequency, and (d) the plasma rotation frequency at the $q=2$ and $q=3$ surfaces. The dashed vertical lines in (a), (b), and (d) indicate the moment of time when the non-linear coupling between the mode and the plasma flow is switched on. The dashed vertical lines in (c) indicate the location of the $q=2$ and 3 rational surfaces, respectively. The numbered lines in (c) correspond to time: $1-5.21 \times 10^{4} \tau_{A}, 2-5.13$ $\times 10^{4} \tau_{A}, 3-5.03 \times 10^{4} \tau_{A}$, and $4-4.95 \times 10^{4} \tau_{A}$. Both the electromagnetic and the NTV torques are included in this simulation. 
in which the momentum source does not explicitly enter into the equation.

We use the homogeneous Neumann (Dirichlet) boundary condition for $\Delta L$ at the plasma center (edge). The Dirichlet edge boundary condition is motivated by discussions from Ref. 21.

In MARS-Q, the $n=1$ single fluid MHD equations, the vacuum and wall equations, as well as the $n=0$ toroidal momentum balance equation are solved together as an initial value problem. An adaptive, semi-implicit time stepping scheme is employed. ${ }^{11}$ In particular, a fully implicit scheme is used for solving the MHD equations, allowing a time step larger than the Alfvén time without introducing numerical instability. The initial condition for the $n=1$ perturbation is chosen as the eigenfunction of the linear RWM, computed by running the eigenvalue code MARS-F. ${ }^{22}$

\section{NUMERICAL RESULTS}

\section{A. Equilibrium}

We consider a toroidal plasma representing the so called advanced tokamak, with the plasma boundary shape, shown in Fig. 1, resembling a typical JET plasma (in terms of aspect ratio, elongation, and triangularity). The plasma boundary is up-down symmetric. The vacuum toroidal field, at the magnetic axis $R_{0}=2.90 \mathrm{~m}$, is assumed to be $B_{0}=1.22 \mathrm{~T}$. The total plasma current is $1.56 \mathrm{MA}$.

The radial profiles of the equilibrium quantities are shown in Fig. 2. The advanced tokamak aims at high beta, high fraction of non-inductive current drive. A peaked pressure profile, as shown in Fig. 2(b), results in a large fraction of the bootstrap current in the middle region of the plasma column, which in turn creates a rather flat current profile and a slightly reversed $q$ profile in the plasma core. The equilibrium with a broad current profile and a peaked pressure profile tends to be more susceptible to the external kink instability, ${ }^{23}$ which becomes a RWM in the presence of a

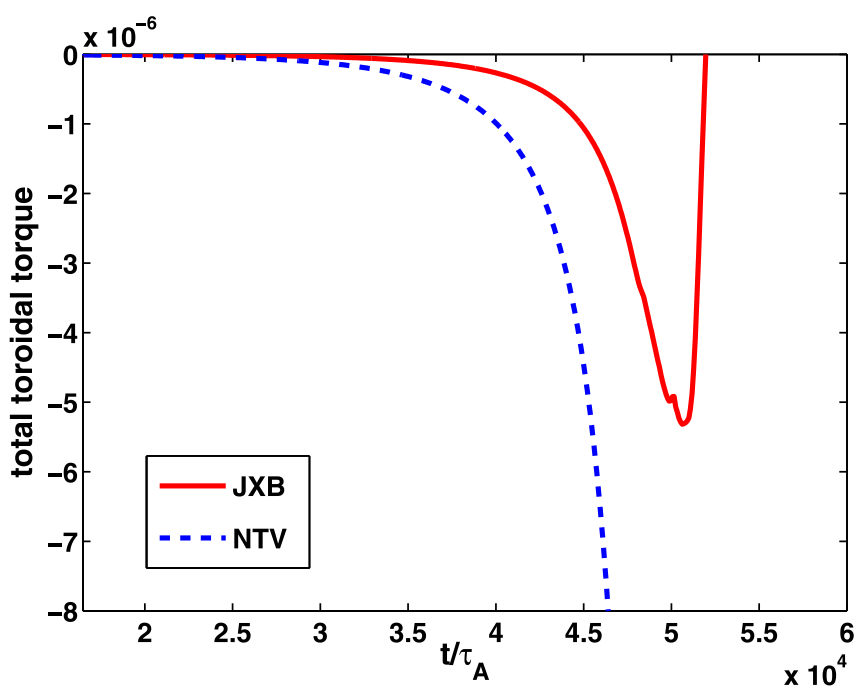

FIG. 5. Time trace of the net toroidal electromagnetic (solid line) and NTV (dashed line) torques acting on the plasma during the non-linear evolution of an initially unstable RWM as described in Fig. 4. close fitting resistive wall. This motivates our choice of the equilibrium for studying the interaction between the RWM and the plasma flow. We note that this equilibrium pressure does not have a clear edge pedestal. For the pressure driven RWM, the edge transport barrier is not a critical factor.

The density profile, shown in Fig. 2(c), is normalized to unity at the magnetic axis. The amplitude of the density only enters into defining the Alfvén time in the dimensionless, single fluid MHD equations. However, the density amplitude does enter into the NTV calculation (via the collisionality coefficients). In this work, we assume a thermal electron number density of $N_{e 0}=3.09 \times 10^{19} \mathrm{~m}^{-3}$. The thermal (Deuterium) ion density is the same as that of the electrons. This yields the Alfvén time of $\tau_{A} \equiv R_{0} \sqrt{\mu_{0} \rho_{0}} / B_{0}=0.86 \mu \mathrm{s}$ at the magnetic axis. Here, $\rho_{0} \equiv N_{e 0} m_{i}$, and $m_{i}$ is the mass of Deuterium ions.

\section{B. Linear stability}

For the chosen equilibrium profiles, we first investigate the linear stability of the external kink mode, using the MARS-F code. Figure 3(a) shows the computed no-wall (dashed line) and ideal-wall (solid line) growth rates of the mode, while scanning the normalized plasma pressure $\beta_{N}$. Only the amplitude of the plasma pressure is varied during the scan, without changing the radial profile shown in Fig. 2(b). The total plasma current is also fixed. An ideal, conformal wall is located at the $1.25 a$ minor radius. The computed no-wall and ideal-wall beta limits, which correspond to the marginal stability points in Fig. 3(a), are $\beta_{N}^{\text {nw }}=2.56$ and $\beta_{N}^{\text {iw }}=3.74$, respectively.

Replacing the ideal wall by a resistive wall, located at the same minor radius $r_{w}=1.25 a$, we obtain unstable RWMs in the plasma pressure range between the no-wall and the idealwall beta limits. In this study, we choose a representative RWM at $\beta_{N}=3.15$, which is half way between the no-wall and ideal-wall limits. The safety factors are $q_{0}=1.78$ at the

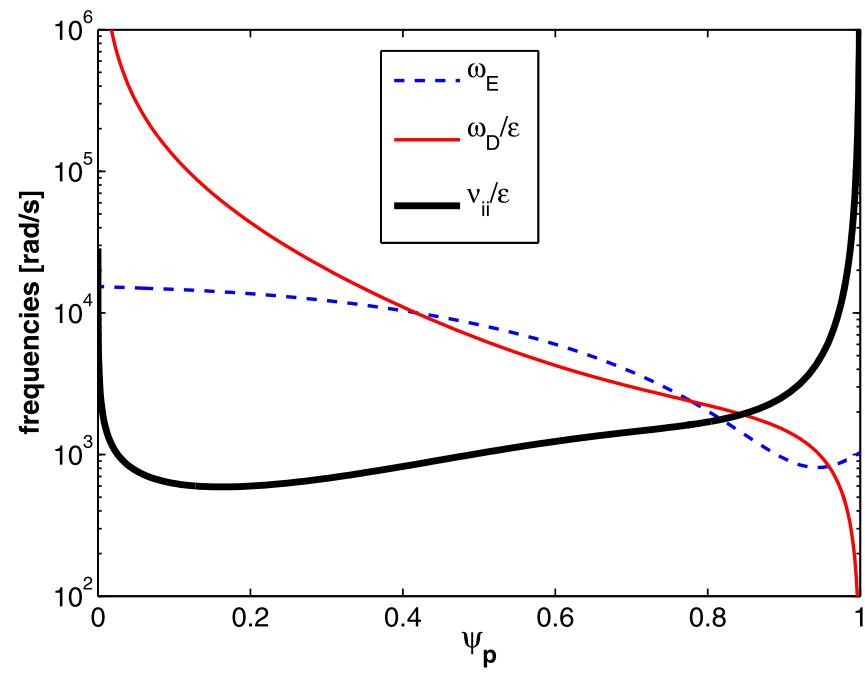

FIG. 6. Radial profiles of the initial (i.e., before closing the non-linear coupling loop) $\mathbf{E} \times \mathbf{B}$ rotation frequency $\omega_{E}$, the precessional drift frequency $\omega_{D}$ of deeply trapped thermal ions at thermal velocity, and the ion-ion collision frequency $\nu_{i i} . \varepsilon$ is approximately the inverse aspect ratio. 
magnetic axis, $q_{a}=3.27$ at the plasma edge, and $q_{95}=2.73$. Assuming a resistive plasma, with the Lundquist number $S=10^{7}$ and a uniform plasma resistivity (a more realistic resistivity profile, e.g., $S \propto T_{e}^{3 / 2}$, tends to increase the TM contribution towards the plasma edge, which is what we try to avoid in this work), and utilizing the parallel sound wave damping model (with $\kappa_{\|}=1.5$ ) as described in Sec. II, we compute the stability of the RWM in the presence of toroidal plasma flow. We scan the amplitude of the flow speed while fixing the radial profile of the toroidal rotation as shown in Fig. 2(d). The growth rate of the RWM versus the on-axis rotation frequency $\Omega_{0}$ is reported in Fig. 3(b). Note that we normalize the mode growth rate by the wall time $\tau_{w}$ here. Although a fixed wall time of $\tau_{w}=10^{4} \tau_{A}=8.6 \mathrm{~ms}$ is assumed in these computations, we mention that the growth rate, normalized by the wall time, depends neither on the wall time nor on the Alfvén time, for a typical inertia-free RWM as studied in this case. The RWM is fully stabilized by the plasma flow, when the on-axis rotation frequency exceeds a critical value of $\Omega_{0}^{\mathrm{cr}}=2.73 \times 10^{-2} \omega_{A}$, where $\omega_{A}=B_{0} /\left(R_{0} \sqrt{\mu_{0} \rho_{0}}\right)$ is the on-axis toroidal Alfvén frequency. The damping of the mode in this case comes from the Alfvén and sound wave continuum damping, as well as from the extra ion Landau damping represented by the parallel viscous force term. As long as the plasma flow speed remains sub-sonic, which is the case considered here, more resonant damping is achieved with increasing the rotation speed, explaining the monotonic decrease of the mode's growth rate as shown in Fig. 3(b).

\section{Interaction between initially unstable RWM and flow}

The stability analysis in Fig. 3(b) allows us to choose two representative cases for investigating the interaction between the RWM and the plasma flow. For the first case,
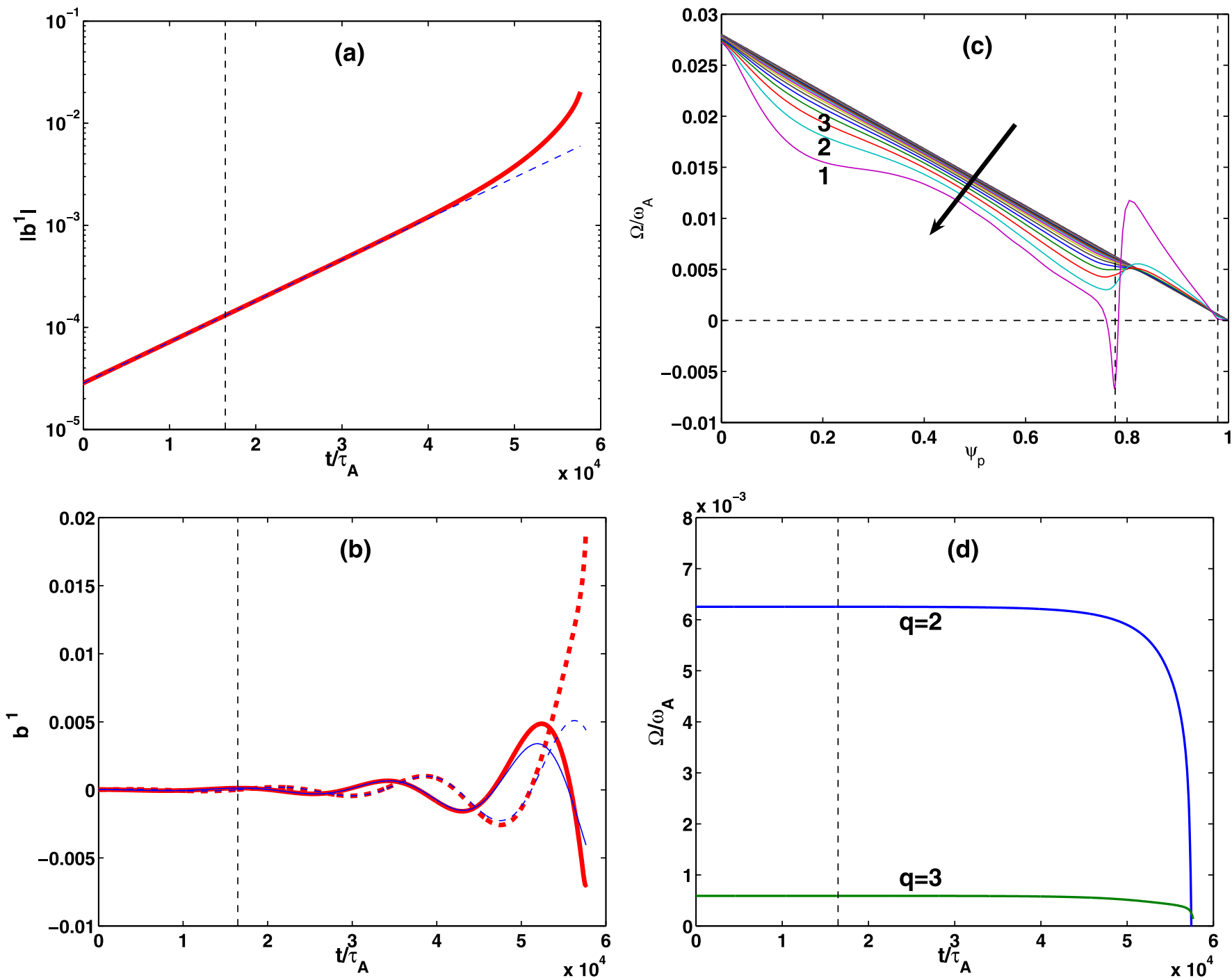

FIG. 7. Evolution of an initially unstable RWM: (a) the amplitude of the perturbed radial field $b^{1}$ at the $q=2$ surface, (b) the real and imaginary parts of the perturbed radial field $b^{1}$ at the $q=2$ surface, (c) the radial profile of the plasma rotation frequency, and (d) the plasma rotation frequency at the $q=2$ and $q=3$ surfaces. The dashed vertical lines in (a), (b), and (d) indicate the moment of time when the non-linear coupling between the mode and the plasma flow is switched on. The dashed vertical lines in (c) indicate the location of the $q=2$ and 3 rational surfaces, respectively. The numbered lines in (c) correspond to time: $1-5.77 \times 10^{4} \tau_{A}, 2-5.67 \times 10^{4} \tau_{A}$, and $3-5.58 \times 10^{4} \tau_{A}$. Only the electromagnetic is included in this simulation. 


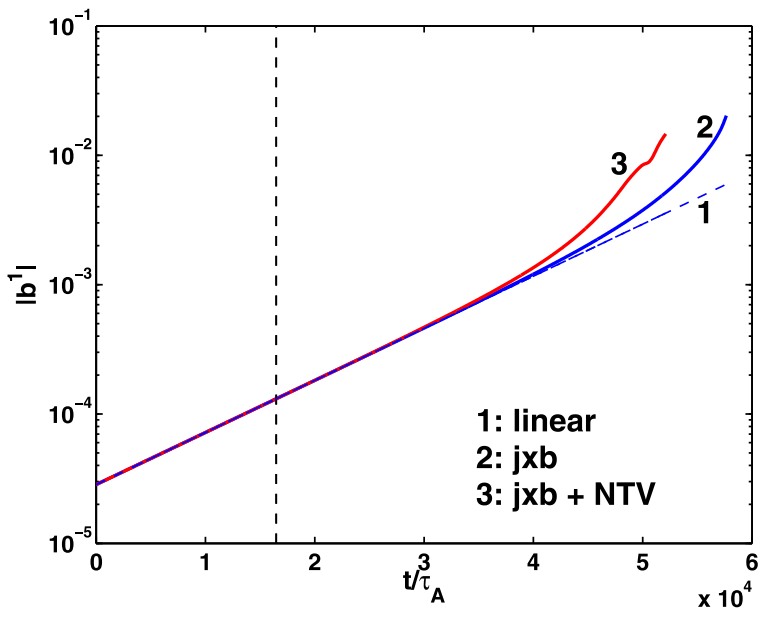

FIG. 8. Time traces of the amplitude of the perturbed radial field $b^{1}$ at the $q=2$ surface, with and without inclusion of the NTV torque in the simulation. The dashed curve corresponds to the exponential growth of the initially unstable linear mode. The dashed vertical line indicates the moment of time when the non-linear coupling between the mode and the plasma flow is switched on. reported in this subsection, we consider an initial plasma rotation frequency of $\Omega_{0}=2.5 \times 10^{-2} \omega_{A}$. In this case, the RWM is still linearly unstable. We launch the MARS-Q simulation starting from the computed linear eigenfunction at $\Omega_{0}=2.5 \times 10^{-2} \omega_{A}$. The amplitude of the eigenfunction is scaled down to a small value, such that the perturbed radial field amplitude is $\left|b^{1}\right|=3 \times 10^{-5}$ at the $q=2$ rational surface and at the outboard mid-plane.

Here, $b^{1}$ is defined as $b^{1}=J \mathbf{B} \cdot \nabla s$, with $J=(\nabla s \cdot \nabla \chi$ $\times \nabla \phi)^{-1}$ being the jacobian of the curve-linear flux coordinate system $(s, \chi, \phi) . s=\sqrt{\psi_{p}}$ is the square root of the normalized equilibrium poloidal flux $\psi_{p}\left(\psi_{p}=0\right.$ corresponds to the magnetic axis, and $\psi_{p}=1$ corresponds to the plasma edge). $\chi$ is the (generalized) poloidal angle. The perturbed magnetic field quantities are normalized by the on axis vacuum toroidal field $B_{0}$. The $b^{1}$ field reported in this study is always taken at the outboard mid-plane.

Figure 4 summarizes the computational results. In order to verify whether the linear phase of the initial value code (MARS-Q) recovers the results of the eigenvalue solver
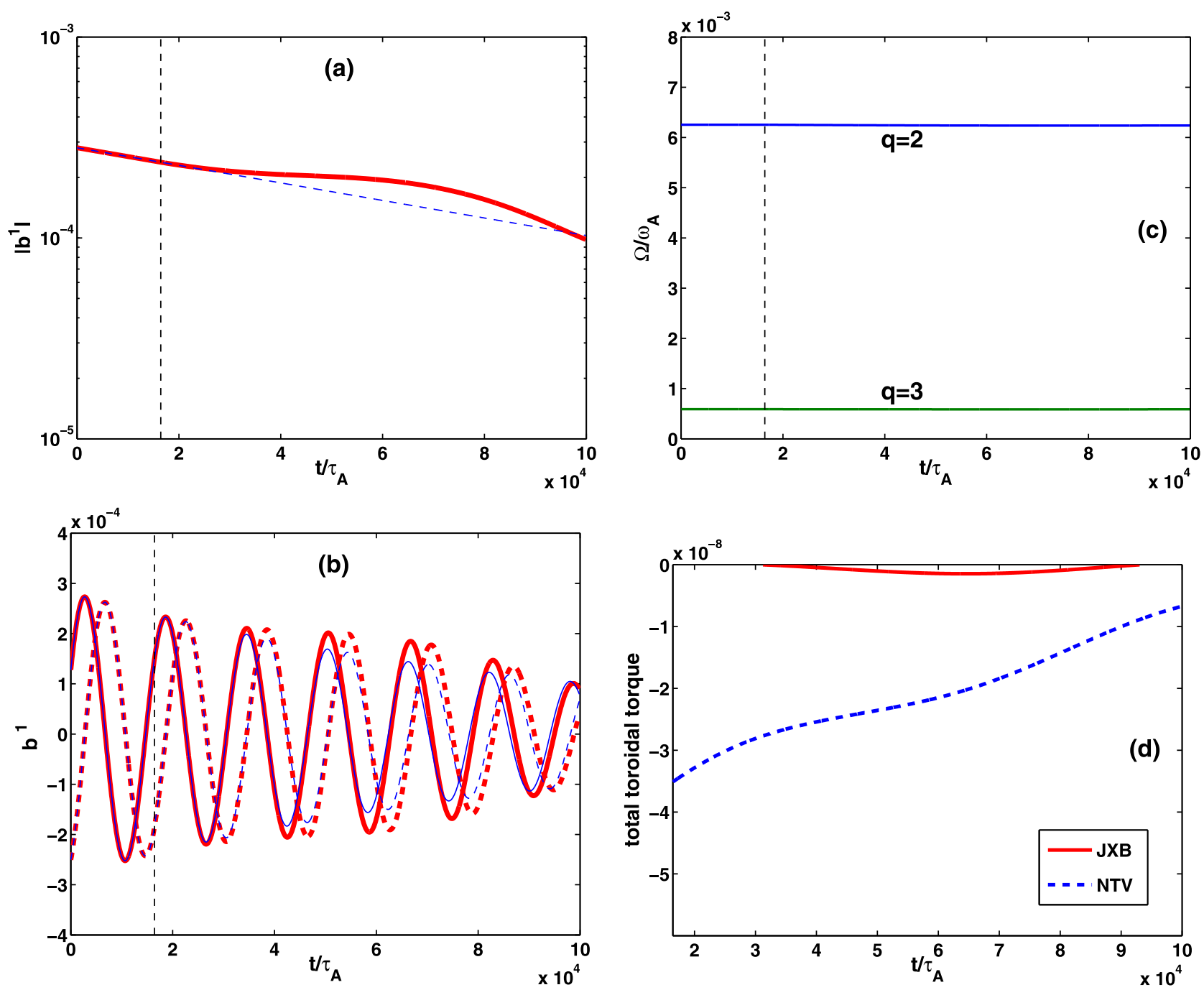

FIG. 9. Evolution of an initially stable RWM: (a) the amplitude of the perturbed radial field $b^{1}$ at the $q=2$ surface, (b) the real and imaginary parts of the perturbed radial field $b^{1}$ at the $q=2$ surface, (c) the plasma rotation frequency at the $q=2$ and $q=3$ surfaces, and (d) the net toroidal electromagnetic and NTV torques acting on the plasma. The dashed vertical lines in (a), (b), and (c) indicate the moment of time when the non-linear coupling between the mode and the plasma flow is switched on. The initial mode amplitude, normalized by $B_{0}$, is $\left|b^{1}\right|(q=2)=2.8 \times 10^{-4}$. 
(MARS-F), we decouple the time evolution of the RWM from the momentum solver (i.e., the initial toroidal rotation is not changed) for the first 280 time steps, which ends at time $T_{1}=1.65 \times 10^{4} \tau_{A}=14 \mathrm{~ms}, \tau_{A}=1 / \omega_{A}$, indicated by the vertical dashed lines in Figs. 4(a), 4(b), and 4(d). The solid curve in Fig. 4(a) shows the amplitude of the radial field $b^{1}$ at the $q=2$ surface, computed by MARS-Q. Indeed, an exponential growth is recovered during $t \in\left[0, T_{1}\right]$. An analytic fitting of the numerical data yields $\gamma \tau_{A}=9.27 \times 10^{-5}+3.58 \times 10^{-4} i$ for the linear phase, agreeing well with the eigenvalue of $\gamma \tau_{A}=9.63 \times 10^{-5}$ $+3.58 \times 10^{-4} i$ from the eigenvalue solver MARS-F. Note that, because of the plasma flow, the mode is rotating while growing. Figure 4(b) shows both the real (thick solid curve) and imaginary (thick dashed curve) parts of $b^{1}(q=2)$. The analytic fitting, shown as dashed curve in Fig. 4(a) and thin-line curves in Fig. 4(b), is performed for these complex quantity data.
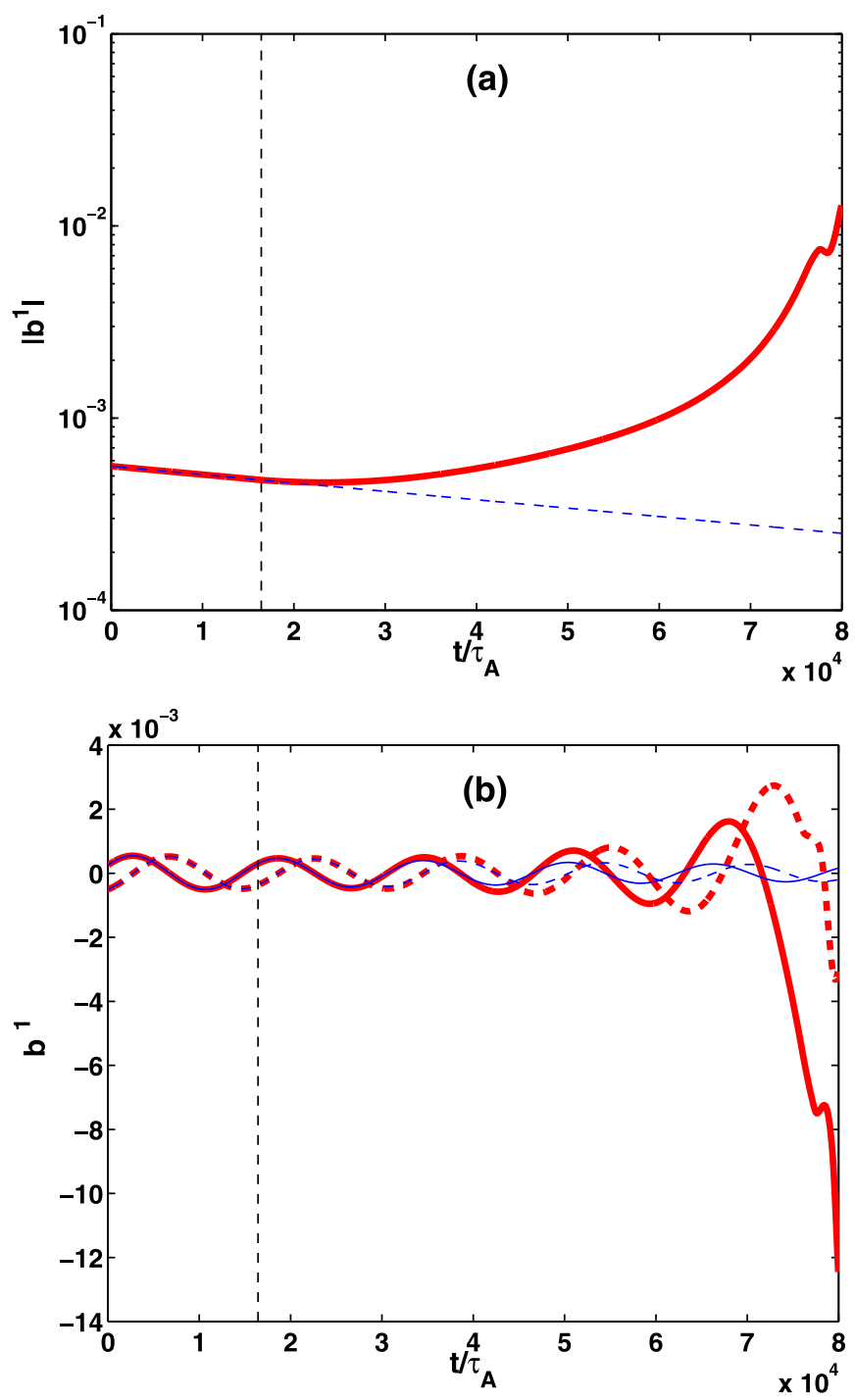

At time $t=T_{1}$, we turn on the non-linear coupling between the RWM and the plasma flow, and continue the MARS-Q simulation. In the momentum balance equation, both the electromagnetic and the NTV torques are included. During this phase, the mode becomes more unstable than the linear instability, accompanied by a flow damping shown in Figs. 4(c) and 4(d). Figure 4(c) shows the evolution of the radial profile of the toroidal rotation frequency during the nonlinear phase. In this and later simulations, we plot the radial profiles only at about 60 time slices, equally spaced in time. The total number of time steps for this simulation is 855 (at $t=5.22 \times 10^{4} \tau_{A}$ ), before a full braking of the rotation occurs. Figure 4(d) plots the simulated time traces of the rotation frequency at the $q=2$ and $q=3$ rational surfaces. We notice that the rotation also switches sign near the end of the simulation. This is mainly due to the momentum sink induced by the NTV torque, as will be shown in further analysis. The time interval between $T_{1}$ and the full braking
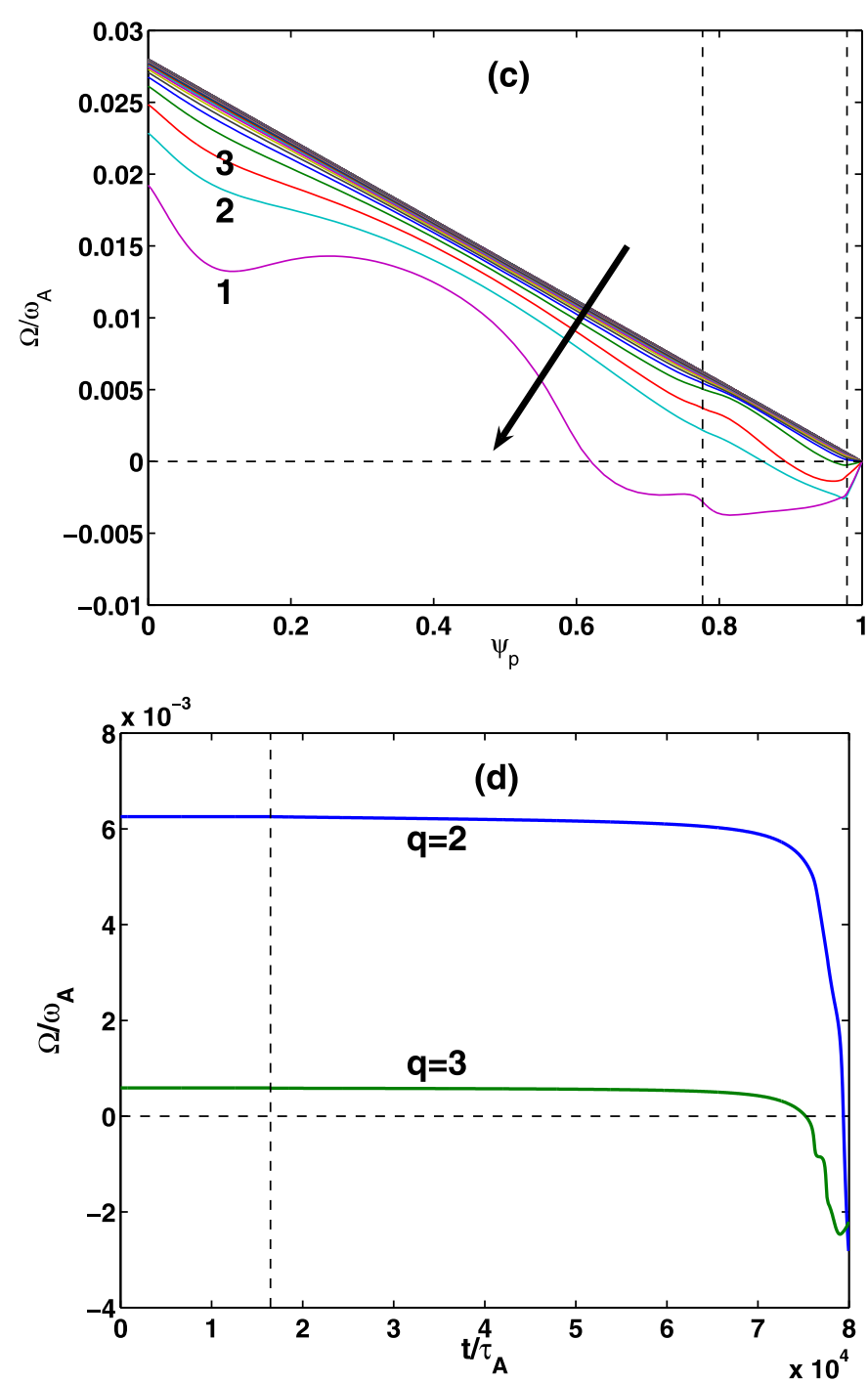

FIG. 10. Evolution of an initially stable RWM: (a) the amplitude of the perturbed radial field $b^{1}$ at the $q=2$ surface, (b) the real and imaginary parts of the perturbed radial field $b^{1}$ at the $q=2$ surface, (c) the radial profile of the plasma rotation frequency, and (d) the plasma rotation frequency at the $q=2$ and $q=3$ surfaces. The dashed vertical lines in (a), (b), and (d) indicate the moment of time when the non-linear coupling between the mode and the plasma flow is switched on. The dashed vertical lines in (c) indicate the location of the $q=2$ and 3 rational surfaces, respectively. The numbered lines in (c) correspond to time: $1-8.00 \times 10^{4} \tau_{A}, 2-7.86 \times 10^{4} \tau_{A}$, and $3-7.73 \times 10^{4} \tau_{A}$. The initial mode amplitude, normalized by $B_{0}$, is $\left|b^{1}\right|(q=2)=5.6 \times 10^{-4}$. Both the electromagnetic and the NTV torques are included in this simulation. 
of the edge rotation (beyond the $q=2$ surface) of the plasma is about $3.4 \times 10^{4} \tau_{A}=29 \mathrm{~ms}$. The time period for a considerable change of the rotation, as well as the toroidal torques shown in Fig. 5, is about $10 \mathrm{~ms}$ for the given plasma parameters studied here. For a comparison, the full braking time constant of the toroidal plasma flow is reported to be about $20 \mathrm{~ms}$ for a high-beta DIII-D plasma, due to the onset of the RWM. $^{5}$ The rotation braking time, observed in typical MAST plasmas using the resonant magnetic perturbation fields, was also reported to be tens of milliseconds. ${ }^{24}$

The rotation braking shown in Figs. 4(c) and 4(d) is associated with the momentum sink terms-the electromagnetic and the NTV torques in our model. Figure 5 compares time traces of the radially integrated, net $\mathbf{j} \times \mathbf{b}$ and NTV torques acting on the plasma column, during the non-linear phase of the MARS-Q simulation, for the same case shown in Fig. 4. These net torques have negative values, indicating deceleration of the plasma flow. More interestingly, we find that, by amplitude, the net NTV torque is much larger than the net electromagnetic torque. In other words, the NTV torque contributes the major part of rotational damping in this case. In fact, this holds for all the cases considered in this paper. However, we cannot conclude that the NTV torque is always dominant over the $\mathbf{j} \times \mathbf{b}$ for damping the plasma flow in a toroidal plasma. One counter example was reported in Ref. 24, where a static RMP (instead of a nearly static MHD mode) field was applied to a MAST plasma, and where we observed a larger electromagnetic torque than the NTV torque for braking the plasma flow. There are at least two factors that affect the comparison between these two sink terms. One is the number of rational surfaces inside the plasma. In the case reported in Ref. 24, the applied field has a high- $n(n=3,4,6)$ toroidal mode number and high $q$-value, resulting in a very large number of
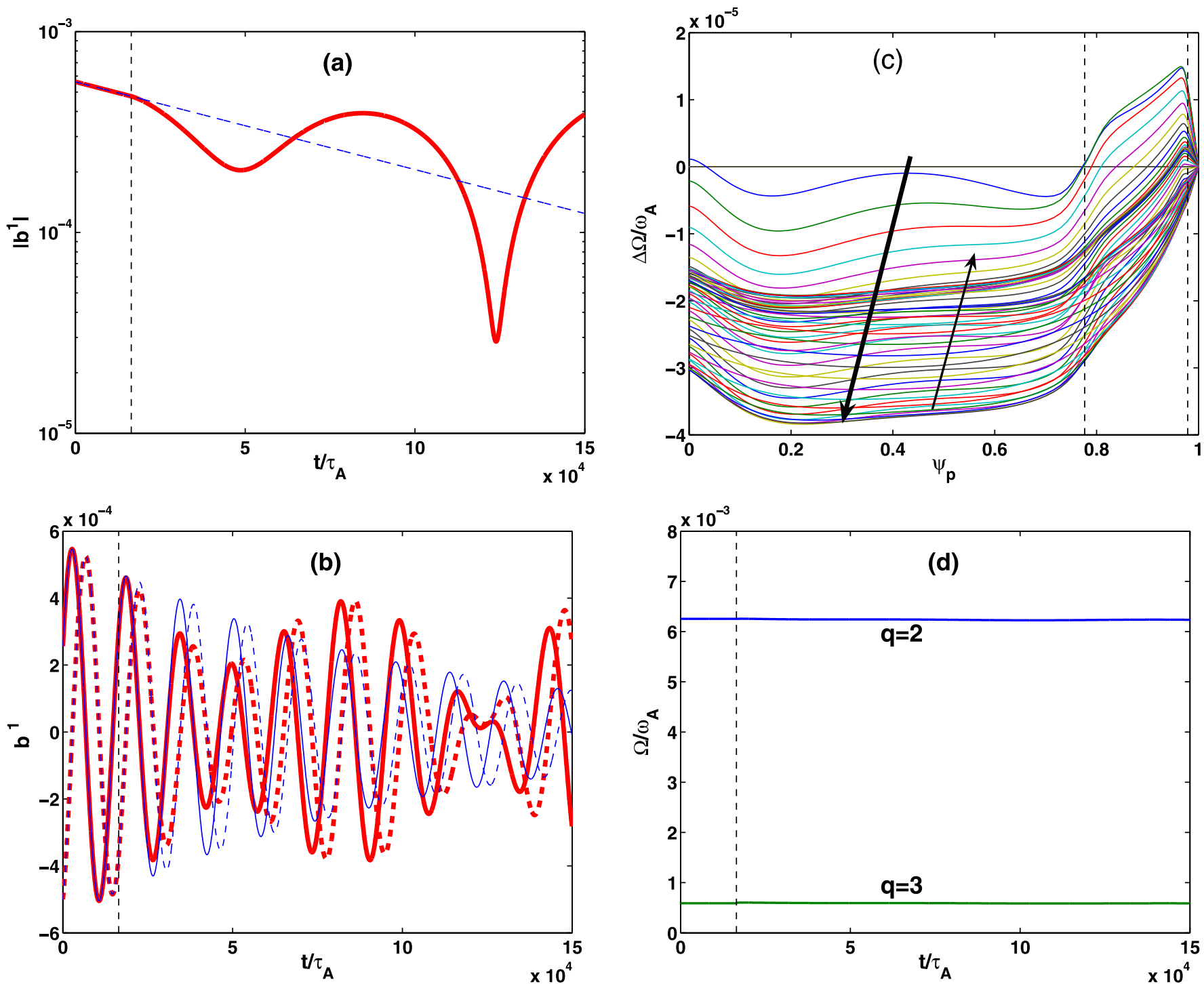

FIG. 11. Evolution of an initially stable RWM: (a) the amplitude of the perturbed radial field $b^{1}$ at the $q=2$ surface, (b) the real and imaginary parts of the perturbed radial field $b^{1}$ at the $q=2$ surface, (c) the radial profile of the change of the plasma rotation frequency, and (d) the plasma rotation frequency at the $q=2$ and $q=3$ surfaces. The dashed vertical lines in (a), (b), and (d) indicate the moment of time when the non-linear coupling between the mode and the plasma flow is switched on. The dashed vertical lines in (c) indicate the location of the $q=2$ and 3 rational surfaces, respectively. The initial mode amplitude, normalized by $B_{0}$, is $\left|b^{1}\right|(q=2)=5.6 \times 10^{-4}$. Only the electromagnetic torque is included in this simulation. 
rational surfaces. This facilitates generation of large net electromagnetic torque. The second factor is the initial plasma flow speed. At fast plasma flow (as that in low aspect ratio tokamaks such as MAST), the NTV torque tends to be small. The NTV torque becomes considerably larger at slow plasma flow, due to a resonant enhancement effect as discussed in Ref. 24 and references therein.

We also note that the amplitudes of both the net electromagnetic and the NTV torques, shown in Fig. 5, grow faster than the mode amplitude. In fact, during the initial phase of the non-linear interaction, say between $t_{1}=T_{1}$ and $t_{2}=3 \times 10^{4} \tau_{A}$ when the mode still grows nearly linearly, the amplitudes of the net torques also grow nearly linearly, with the numerically recovered growth rates of about $\gamma_{j x b} \tau_{A}=1.83 \times 10^{-4}$ and $\gamma_{N T V} \tau_{A}=1.88 \times 10^{-4}$ for the net $\mathbf{j} \times \mathbf{b}$ and NTV torques, respectively. These growth rates are about twice larger than that of the linear RWM, confirming the quadratic nature of the torques.

The amplitudes of the torques shown in Fig. 5 can be converted into the SI unit by multiplying the dimensionless results by a factor $R_{0}^{3} B_{0}^{2} / \mu_{0}=1.3 \times 10^{7}$, yielding total torques of up to tens of $\mathrm{Nm}$ for this case. We point out that, since both the electromagnetic and the NTV torques are quadratic functions of the mode amplitude, for an unstable RWM, the torque can become large as the mode amplitude grows to a large value. For a stable RWM at smaller amplitude, both torques can be in order of magnitude smaller as shown in Subsection III D. Several Nm's torque is measured in JET plasmas by applying an $n=1$ RMP fields. ${ }^{25}$ Dedicated experiments have also been carried out in DIII-D, ${ }^{26}$ where the measured NTV torque is in the order of several Nm. The direct modeling of this DIII-D plasma using MARS-Q also recovers well the experimental value. $^{24}$

Figure 6 shows important frequencies relevant to the NTV torque calculation, for the plasma studied in this work. In the core region, both the ion-ion collision frequency and the $\mathbf{E} \times \mathbf{B}$ frequency (shown at the initial stage) are small compared to the precessional drift frequency, resulting in a predominantly resonant NTV torque in the so-called superbanana or superbanana plateau regime. This resonant torque can be large and is responsible for the fast decay of rotation in the plasma core, as shown in Fig. 4(c). In the middle of the plasma column, the $\mathbf{E} \times \mathbf{B}$ rotation frequency is larger than the precession and collision frequencies of trapped ions. The resulting NTV torque has a predominant non-resonant component (in the so-called $\nu-\sqrt{\nu}$ regime), which is normally small. In the region close to the plasma edge, where $\omega_{E}$ is comparable or smaller than $\omega_{D} / \epsilon$, we expect again a resonant NTV torque which is larger. Very close to the plasma edge, the collision frequency dominates resulting in the so-called $1 / \nu$-regime, where the NTV torque is again relatively small.

So far, most of the theory ${ }^{6-8}$ on the rotational damping due to the onset of the RWM has only included the electromagnetic torque as the momentum sink. We perform a similar simulation by excluding the NTV torque from the momentum balance equation. The results are summarized in Fig. 7 for the unstable RWM case at $\Omega_{0}(t=0)=2.5 \times 10^{-2} \omega_{A}$. The electromagnetic torque alone is also capable of braking the flow, but the effect is generally more localized near rational surfaces. Similar to the previous case (see Fig. 4(d)), there is a narrow time window, during which the flow is rapidly damped, as shown in Fig. 7(d).

In the absence of the NTV torque, the non-linear growth of the RWM becomes slower during the rotation braking phase, as shown in Fig. 8. This is expected to be taken into account a slower damping of the flow.

\section{Interaction between initially stable RWM and flow}

Perhaps, a practically more important problem is the interaction between a (marginally) stable RWM and the plasma flow. It has been shown, in both experiments and theory, ${ }^{1}$ that the RWM often stays marginally stable even in the presence of a strong damping, either from the kinetic damping or other damping mechanisms. One example is shown in Fig. 3(b) with a strong parallel sound wave damping. A shallowly stable RWM is sensitive to external field perturbations, often leading
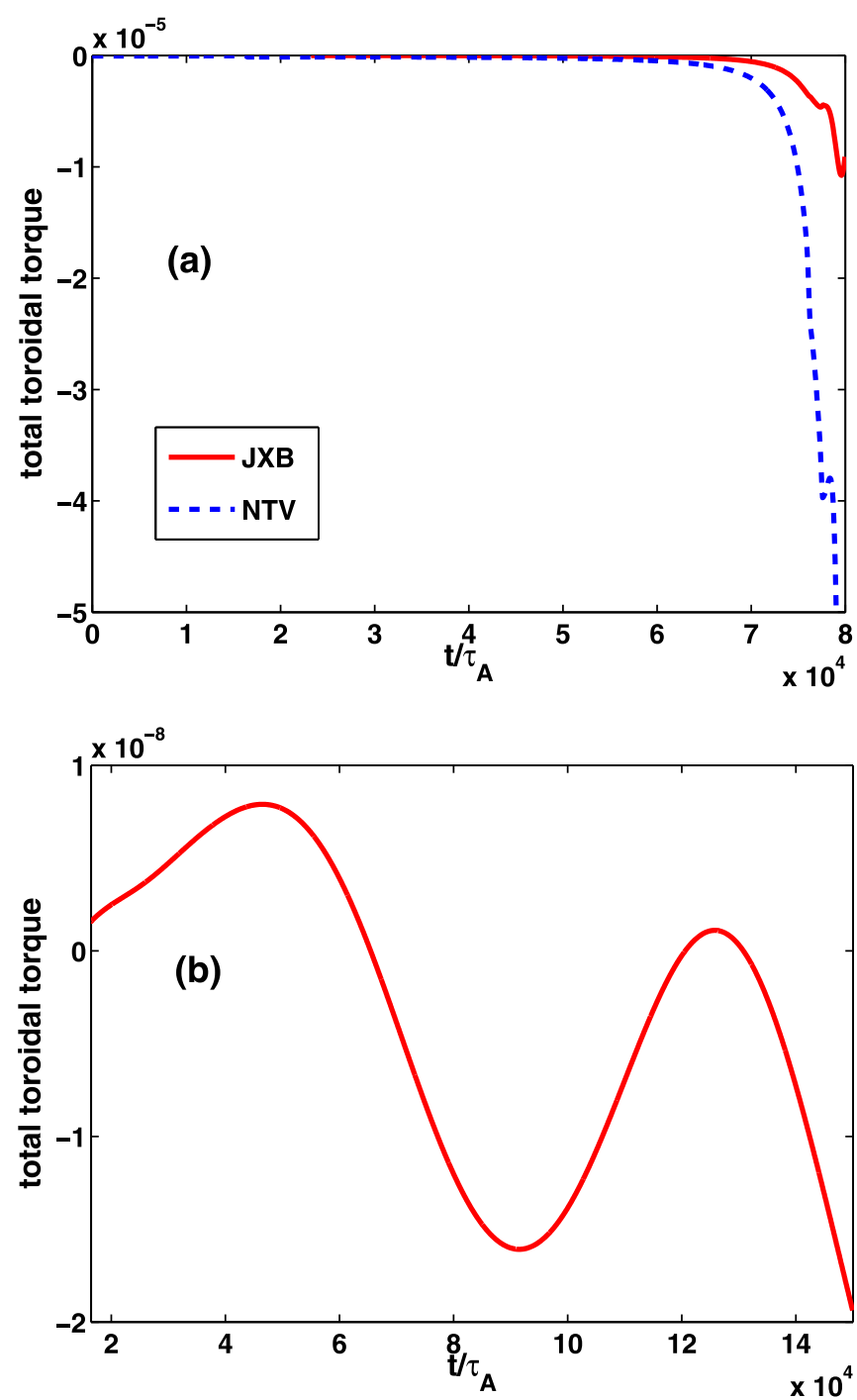

FIG. 12. Time traces of the net toroidal electromagnetic (solid line) and NTV (dashed line) torques acting on the plasma, during the non-linear evolution of an initially stable RWM, with the initial mode amplitude $\left|b^{1}\right|(q=2)=5.6 \times 10^{-4}$ : (a) both electromagnetic and NTV torques are included in the toroidal torque balance and (b) only the electromagnetic torque is included. 
to the so called resonant field amplification effect, ${ }^{27}$ which in turn brakes the plasma flow. We investigate this phenomenon in this subsection. In particular, we study how the flow braking is affected by assuming a different level of the initial (stable) mode amplitude. We assume an initial rotation frequency of $\Omega_{0}(t=0)=2.8 \times 10^{-2}$, which stabilizes the RWM according to Fig. 3(b).

Figure 9 reports a first case, where we assume a very small initial mode amplitude, $\left|b^{1}\right|(q=2, t=0)=2.8 \times 10^{-4}$. Again, the linear phase is well recovered by the initial value solver, during $t \in\left[0, T_{1}=1.65 \times 10^{4} \tau_{A}\right]$. When the non-linear coupling between the mode and the plasma flow is introduced at time $T_{1}$, no appreciable braking of the flow is observed, in the presence of both electromagnetic and NTV torques.

However, increasing the initial mode amplitude by a factor of $2,\left|b^{1}\right|(q=2, t=0)=5.6 \times 10^{-4}$, results in a full braking of the plasma flow and the eventual onset of an unstable RWM, as demonstrated by Fig. 10. More interestingly, by excluding the contribution of the NTV torque from the momentum balance equation and keeping the same initial mode amplitude, only a slight braking of the flow is observed, and the mode stays stable after a rather long simulation time $\left(T_{\text {end }}=1.5 \times 10^{5} \omega_{A}\right.$ after 2520 time steps), as shown by Fig. 11. Note that Fig. 11(c) shows the change of the rotation frequency $\Delta \Omega$, which is non-monotonic, with the radial profile evolution shown by two arrows. The change is small compared to the initial rotation frequency. But nevertheless, this small change of rotation, in particular its radial profile, does impact the mode evolution as shown in Figs. 11(a) and 11(b). The global radial distribution of $\Delta \Omega$, at later stage of the simulation, is achieved due to the momentum diffusion. The faster-than-linear decrease of the mode amplitude, during certain periods of time, is associated with the change of the radial profile of flow, and consequently,
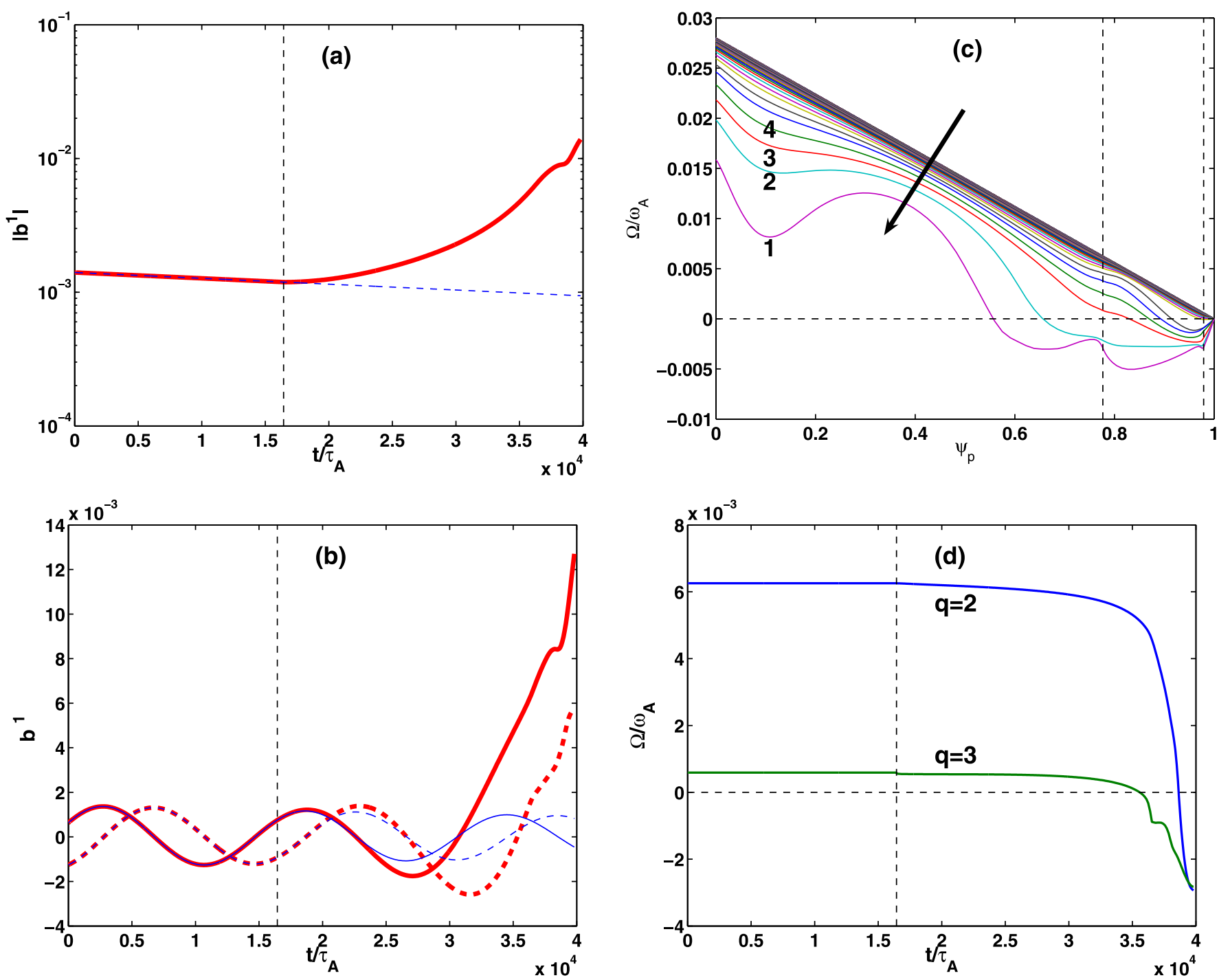

FIG. 13. Evolution of an initially stable RWM: (a) the amplitude of the perturbed radial field $b^{1}$ at the $q=2$ surface, (b) the real and imaginary parts of the perturbed radial field $b^{1}$ at the $q=2$ surface, (c) the radial profile of the plasma rotation frequency, and (d) the plasma rotation frequency at the $q=2$ and $q=3$ surfaces. The dashed vertical lines in (a), (b), and (d) indicate the moment of time when the non-linear coupling between the mode and the plasma flow is switched on. The dashed vertical lines in (c) indicate the location of the $q=2$ and 3 rational surfaces, respectively. The numbered lines in (c) correspond to time: $1-3.98 \times 10^{4} \tau_{A}, 2-3.91 \times 10^{4} \tau_{A}, 3-3.85 \times 10^{4} \tau_{A}$, and $4-3.79 \times 10^{4} \tau_{A}$. The initial mode amplitude, normalized by $B_{0}$, is $\left|b^{1}\right|(q=2)=1.4 \times 10^{-3}$. Both the electromagnetic and the NTV torques are included in this simulation. 
the deviation of the mode structure from that of the linear eigenmode.

A comparison of the net electromagnetic versus NTV torque, shown in Fig. 12(a), again confirms that the latter contributes the dominant momentum sink. In the absence of the NTV torque (Fig. 12(b)), the electromagnetic torque is too small to give an appreciable effect on the flow damping.

Further increase of the initial mode amplitude leads to a full rotation braking with or without the NTV contributions. Figures 13 and 14 show one example, where the initial mode amplitude is increased by 5 times compared to that of Fig. 9. Figures 15(a) and 15(b) compare time traces of the mode amplitude $\left|b^{1}\right|(q=2)$ with or without the NTV torque, for two different values of the initial mode amplitude.

As a final comparison, we plot time traces of the mode amplitude and the rotation frequencies at rational surfaces, in Figs. 16(a) and 16(b), respectively. The initial mode amplitude is increased by factors of $2,5,10$, starting from the lowest level of $\left|b^{1}\right|(q=2, t=0)=2.8 \times 10^{-4}$. Both electromagnetic and NTV torques are included here. We observe a progressively earlier time of the rotational braking and the mode onset, as the initial mode amplitude is increased. The critical mode amplitude, above which the non-linearly coupled system bifurcates from the stable solution to an unstable solution, is between $1.4-2.8 \times 10^{-4}$ for $\left|b^{1}\right|(q=2)$ at $t=0$, for the toroidal plasma considered in this work.

\section{CONCLUSION AND DISCUSSION}

We have presented toroidal simulation results of the non-linear interaction between the RWM and the toroidal plasma flow, for a typical advanced tokamak plasma. By tuning the amplitude of the initial plasma flow, the linear RWM, from which we launch the non-linear simulation, can be either stable or unstable. An initially unstable RWM brakes the plasma flow, leading to a non-linearly more
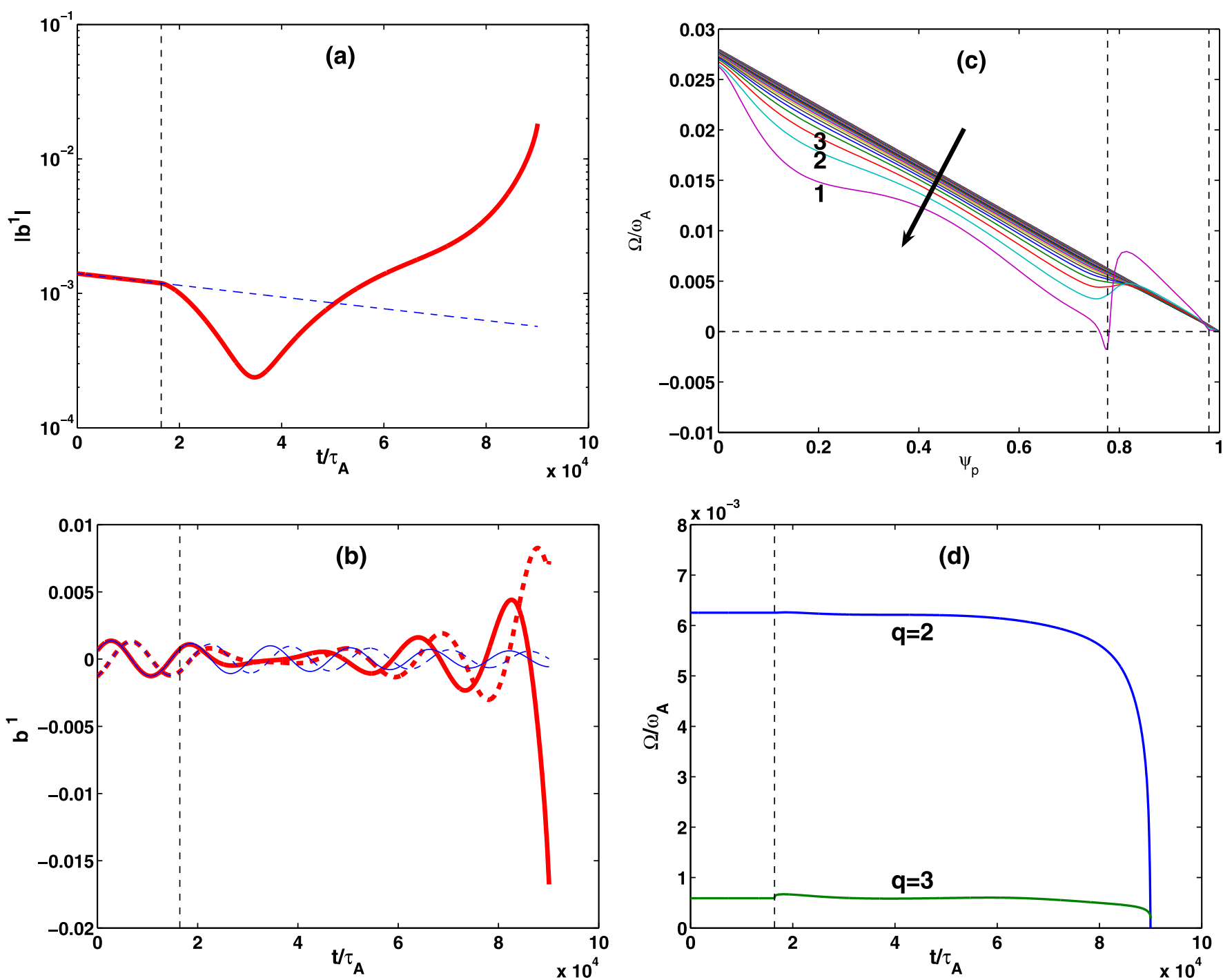

FIG. 14. Evolution of an initially stable RWM: (a) the amplitude of the perturbed radial field $b^{1}$ at the $q=2$ surface, (b) the real and imaginary parts of the perturbed radial field $b^{1}$ at the $q=2$ surface, (c) the radial profile of the plasma rotation frequency, and (d) the plasma rotation frequency at the $q=2$ and $q=3$ surfaces. The dashed vertical lines in (a), (b), and (d) indicate the moment of time when the non-linear coupling between the mode and the plasma flow is switched on. The dashed vertical lines in (c) indicate the location of the $q=2$ and 3 rational surfaces, respectively. The numbered lines in (c) correspond to time: $1-9.00 \times 10^{4} \tau_{A}, 2-8.85 \times 10^{4} \tau_{A}$, and $3-8.70 \times 10^{4} \tau_{A}$. The initial mode amplitude, normalized by $B_{0}$, is $\left|b^{1}\right|(q=2)=1.4 \times 10^{-3}$. Only, the electromagnetic torque is included in this simulation. 

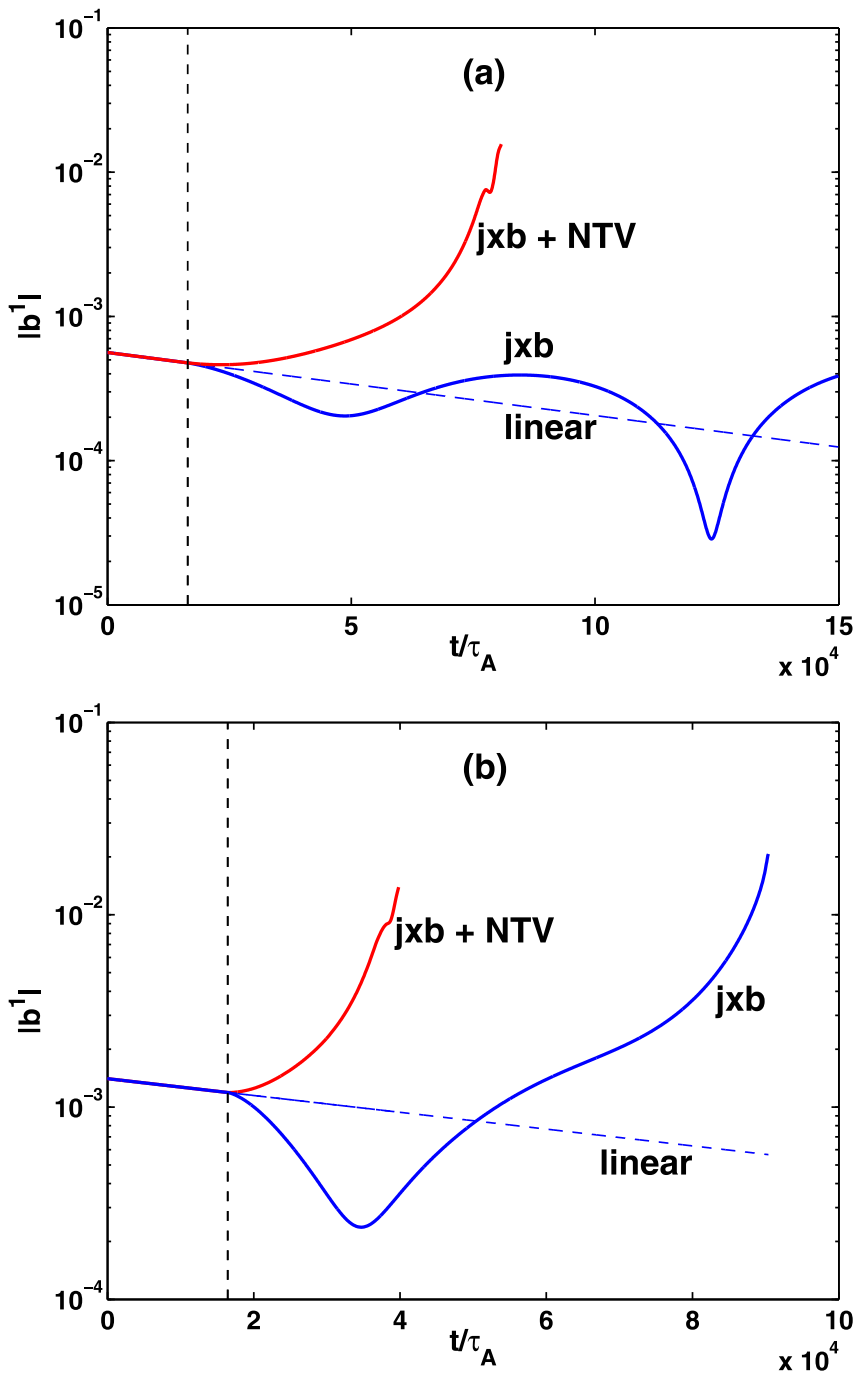

FIG. 15. Time traces of the amplitude of the perturbed radial field $b^{1}$ at the $q=2$ surface, with and without inclusion of the NTV torque in the simulations for an initially stable RWM, with the initial mode amplitude at (a) $\left|b^{1}\right|(q=2)=5.6 \times 10^{-4}$ and (b) $\left|b^{1}\right|(q=2)=1.4 \times 10^{-3}$. The dashed curves correspond to the exponential decay of the initially stable linear mode. The dashed vertical lines indicate the moment of time when the nonlinear coupling between the mode and the plasma flow is switched on.

unstable mode, compared to the linear phase. An initially stable RWM can also brake the flow, due to the resonant amplification effect. Depending on the initial amplitude of the mode perturbation, the non-linear coupling can result in an either stable or unstable solution. The critical (stable) mode amplitude, above which an eventual rotation braking and an unstable mode onset occur, is between 1.4 and $2.8 \times 10^{-4}$ (normalized by the toroidal vacuum field) for the radial field at the $q=2$ surface, for our toroidal example.

The general features of these toroidal simulation results have been qualitatively predicted by cylindrical theory. ${ }^{6,7}$ On the other hand, toroidal simulations provide more quantitative answers, in particular, with regard to the bifurcation amplitude of an initially stable RWM. Also, the change of the radial profile of the flow, which in turn modifies the RWM eigenstructure, is normally not captured by analytic theory. Another interesting observation, which has not been addressed in previous theory, is the dominant role played by
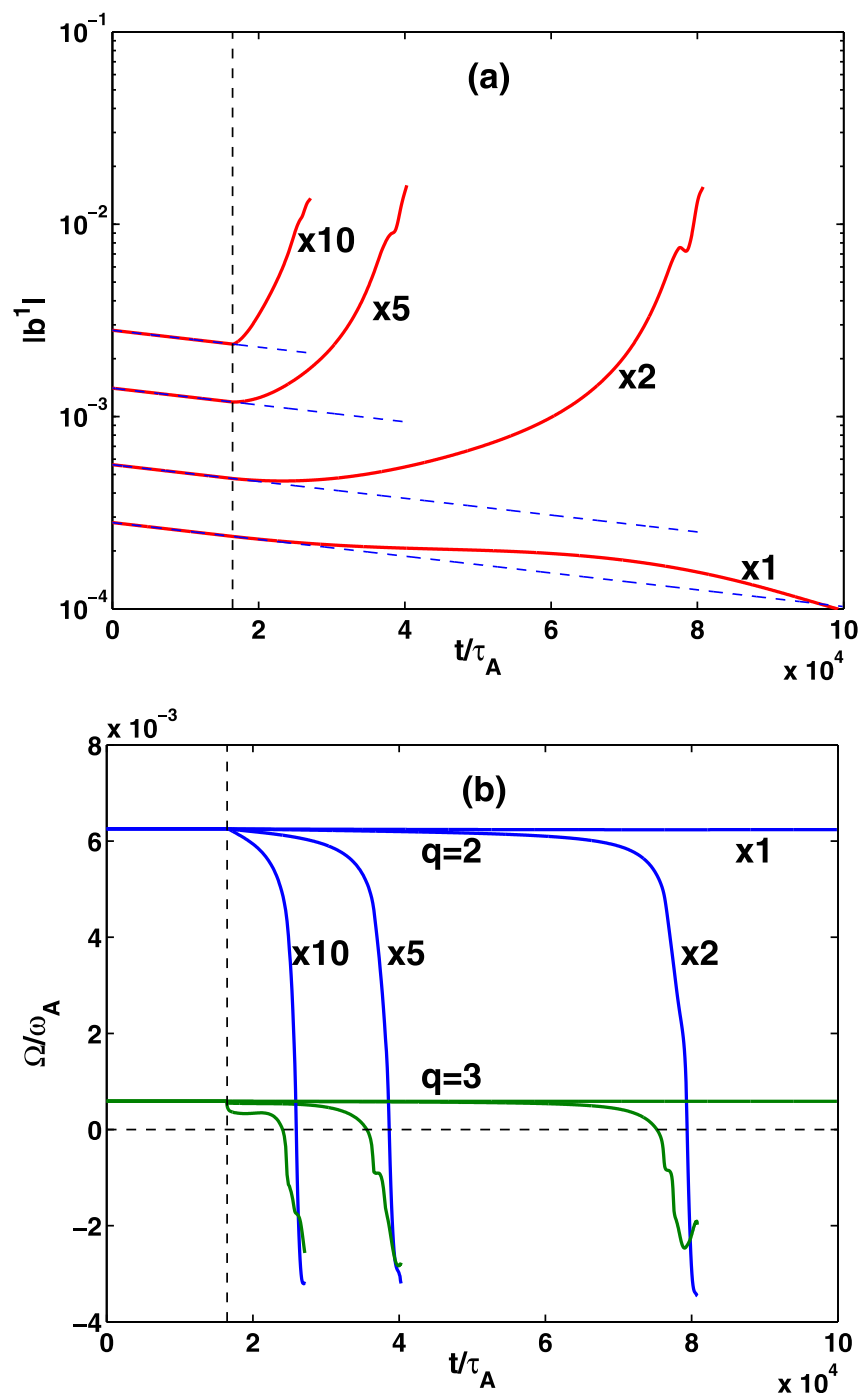

FIG. 16. Time traces of (a) the amplitude of the perturbed radial field $b^{1}$ at the $q=2$ surface, and (b) the plasma rotation frequency at the $q=2$ and $q=3$ surfaces, with various choices of the initial mode amplitude. The dashed curves in (a) correspond to the exponential decay of the initially stable linear mode. The dashed vertical lines indicate the moment of time when the non-linear coupling between the mode and the plasma flow is switched on. Both the electromagnetic and the NTV torques are included in the simulations.

the NTV torque in the flow damping due to the RWM. The net toroidal NTV torque, acting on the plasma due to the presence of the mode as well as a resistive wall, is generally larger than the electromagnetic $\mathbf{j} \times \mathbf{b}$ torque, independent of whether the RWM is initially stable or unstable.

In this work, an initially stable RWM is achieved by a strong parallel sound wave damping, in combination with the Alfvén and sound wave continuum damping, at a sufficiently fast plasma flow. The parallel sound wave damping model is often a crude approximation of the ion Landau damping, but nevertheless helps to suppress the mode, providing interesting toroidal cases for studying the non-linear interaction between the RWM and the plasma flow.

In the future, it is certainly desirable to investigate the RWM-flow coupling with more physics based mode damping models. One such model is the drift kinetic damping included into the MARS-K code. ${ }^{14}$ Because of the often 
rather complicated dependence of the linear mode stability on the plasma flow speed within the kinetic model, we expect generally more rich phenomena in the non-linear coupling between the mode and the plasma rotation braking. In particular, at slow plasma flow, the kinetic effects may introduce significant changes in the time evolution of the rotation profile. This is because, at sub-diamagnetic flow, the precessional drift resonances of trapped particles can become important for the RWM stability. In a self-consistent model, the drift kinetic effect can also modify the eigenfunction of the fluid RWM. This adds even more interesting aspects into the non-linear interaction between the mode and the plasma flow.

As far as the RWM is concerned, there are other interesting aspects that should be addressed in the future work, such as the effects of 3D conducting structures, the presence of electromagnetically thick walls (e.g., the volumetric blanket modules in ITER), the presence of field errors that have been included in cylindrical theory. ${ }^{7,8}$ Finally, quantitative simulations of the RWM interaction with the plasma flow for ITER plasmas can be performed, based on a similar approach as that reported in this work.

\section{ACKNOWLEDGMENTS}

This work was part-funded by The RCUK Energy Programme under Grant No. EP/I501045 and The European Communities under the contract of Association between EURATOM and CCFE. The views and opinions expressed herein do not necessarily reflect those of the European Commission.

Youwen Sun would like to acknowledge the support from the National Magnetic Confinement Fusion Science Program of China under Grant Nos. 2013GB102000 and 2012GB105000 and the National Natural Science Foundation of China under Grant Nos. 11205199 and 10725523.

\footnotetext{
${ }^{1}$ M. S. Chu and M. Okabayashi, Plasma Phys. Controlled Fusion 52, 123001 (2010).

${ }^{2}$ G. Matsunaga, N. Aiba, K. Shinohara, Y. Sakamoto, M. Takechi, T. Suzuki, N. Asakura, A. Isayama, N. Oyama, and JT-60 Team, "Interactions between MHD instabilities in the wall-stabilized high-beta plasmas," in Proceedings of the 23rd IAEA Fusion Energy Conference, EXS/5-3, Daejon, Republic of Korea, 11-16 October 2010.

${ }^{3}$ G. Matsunaga, K. Shinohara, N. Aiba, Y. Sakamoto, A. Isayama, N. Asakura, T. Suzuki, M. Takechi, N. Oyama, H. Urano, and JT-60 Team, Nucl. Fusion 50, 084003 (2010).
}

${ }^{4}$ M. Okabayashi, G. Matsunaga, M. Takechi, J. S. deGrassie, W. W. Heidbrink, Y. In, Y. Q. Liu, E. J. Strait, N. Asakiura, R. Budny, G. Jackson, J. Hanson, R. J. La Haye, M. J. Lanctot, J. Manickam, H. Reimerdes, and K. Shinohara, Phys. Plasmas 18, 056112 (2011).

${ }^{5}$ H. Reimerdes, A. M. Garofalo, E. J. Strait, R. J. Buttery, M. S. Chu, G. L. Jackson, R. J. La Haye, M. J. Lanctot, Y. Q. Liu, J.-K. Park, M. Okabayashi, M. Schaffer, and W. Solomon, Nucl. Fusion 49, 115001 (2009).

${ }^{6}$ R. Fitzpatrick, Phys. Plasmas 9, 3459 (2002).

${ }^{7}$ C. G. Gimblett and R. J. Hastie, Phys. Plasmas 11, 1019 (2004).

${ }^{8}$ R. Fitzpatrick, Phys. Plasmas 14, 022505 (2007).

${ }^{9}$ R. Fitzpatrick and A. Y. Aydemir, Nucl. Fusion 36, 11 (1996).

${ }^{10}$ J. M. Finn, Phys. Plasmas 2, 198 (1995); 2, 3782 (1995).

${ }^{11}$ Y. Liu, A. Kirk, and Y. Sun, "Toroidal modeling of penetration of the resonant magnetic perturbation field," Phys. Plasmas (submitted).

${ }^{12}$ H. Reimerdes, A. M. Garofalo, M. Okabayashi, E. J. Strait, R. Betti, M. S. Chu, B. Hu, Y. In, G. L. Jackson, R. J. La Haye, M. J. Lanctot, Y. Q. Liu, G. A. Navratil, W. M. Solomon, H. Takahashi, R. J. Groebner, and DIII-D Team, Plasma Phys. Controlled Fusion 49, B349 (2007).

${ }^{13}$ M. J. Lanctot, H. Reimerdes, A. M. Garofalo, M. S. Chu, J. M. Hanson, Y. Q. Liu, G. A. Navratil, I. N. Bogatu, Y. In, G. L. Jackson, R. J. La Haye, M. Okabayashi, M. J. Schaffer, O. Schmitz, and E. J. Strait, Phys. Plasmas 18, 056121 (2011).

${ }^{14}$ Y. Liu, M. S. Chu, I. T. Chapman, and T. C. Hender, Phys. Plasmas 15, 112503 (2008).

${ }^{15}$ M. S. Chu, J. M. Greene, T. H. Jensen, R. L. Miller, A. Bondeson, R. W. Johnson, and M. E. Mauel, Phys. Plasmas 2, 2236 (1995).

${ }^{16}$ Y. Liu, A. Bondeson, M. S. Chu, J.-Y. Favez, Y. Gribov, M. Gryaznevich, T. C. Hender, D. F. Howell, R. J. La Haye, and J. B. Lister, Nucl. Fusion 45, 1131 (2005).

${ }^{17}$ J. B. Taylor, Phys. Rev. Lett. 91, 115002 (2003).

${ }^{18}$ Y. Liu, J. W. Connor, S. C. Cowley, C. J. Ham, R. J. Hastie, and T. C. Hender, Phys. Plasmas 19, 102507 (2012).

${ }^{19}$ R. Betti, Phys. Plasmas 5, 3615 (1998).

${ }^{20}$ K. C. Shaing, S. A. Sabbagh, and M. S. Chu, Nucl. Fusion 50, 025022 (2010).

${ }^{21}$ R. Fitzpatrick, Nucl. Fusion 33, 1049 (1993).

${ }^{22}$ Y. Q. Liu, A. Bondeson, C. M. Fransson, B. Lennartson, and C. Breitholtz, Phys. Plasmas 7, 3681 (2000).

${ }^{23}$ A. Bondeson, D.-H. Liu, F. X. Söldner, M. Persson, Yu. F. Baranov, and G. T. A. Huysmans, Nucl. Fusion 39, 1523 (1999).

${ }^{24}$ Y. Q. Liu, A. Kirk, Y. Sun, P. Cahyna, I. T. Chapman, P. Denner, G. Fishpool, A. M. Garofalo, J. R. Harrison, E. Nardon, and MAST Team, Plasma Phys. Controlled Fusion 54, 124013 (2012).

${ }^{25}$ Y. Sun, Y. Liang, H. R. Koslowski, S. Jachmich, A. Alfier, O. Asunta, G. Corrigan, C. Giroud, M. P. Gryaznevich, D. Harting, T. Hender, E. Nardon, V. Naulin, V. Parail, T. Tala, C. Wiegmann, S. Wiesen, and JET-EFDA Contributors, Plasma Phys. Controlled Fusion 52, 105007 (2010).

${ }^{26}$ A. M. Garofalo, W. M. Solomon, J.-K. Park, K. H. Burrell, F. C. DeBoo, M. J. Lanctot, G. R. McKee, H. Reimerdes, L. Schmitz, M. J. Schaffre, and P. B. Snyder, Nucl. Fusion 51, 083018 (2011).

${ }^{27}$ H. Reimerdes, T. C. Hender, S. A. Sabbagh, J. M. Bialek, M. S. Chu, A. M. Garofalo, M. P. Gryaznevich, D. F. Howell, G. L. Jackson, R. J. La Haye, Y. Q. Liu, J. E. Menard, G. A. Navratil, M. Okabayashi, S. D. Pinches, A. C. Sontag, E. J. Strait, W. Zhu, M. Bigi, M. de Baar, P. de Vries, D. A. Gates, P. Gohil, R. J. Groebner, D. Mueller, R. Raman, J. T. Scoville, W. M. Solomon, DIII-D Team, JET-EFDA Contributors, and NSTX Team, Phys. Plasmas 13, 056107 (2006). 